Document downloaded from:

http://hdl.handle.net/10251/141979

This paper must be cited as:

Torregrosa, AJ.; Broatch, A.; Garcia Tiscar, J.; Gómez-Soriano, J. (02-2). Modal decomposition of the unsteady flow field in compression-ignited combustion chambers. Combustion and Flame. 188:469-482. https://doi.org/10.1016/j.combustflame.2017.10.007

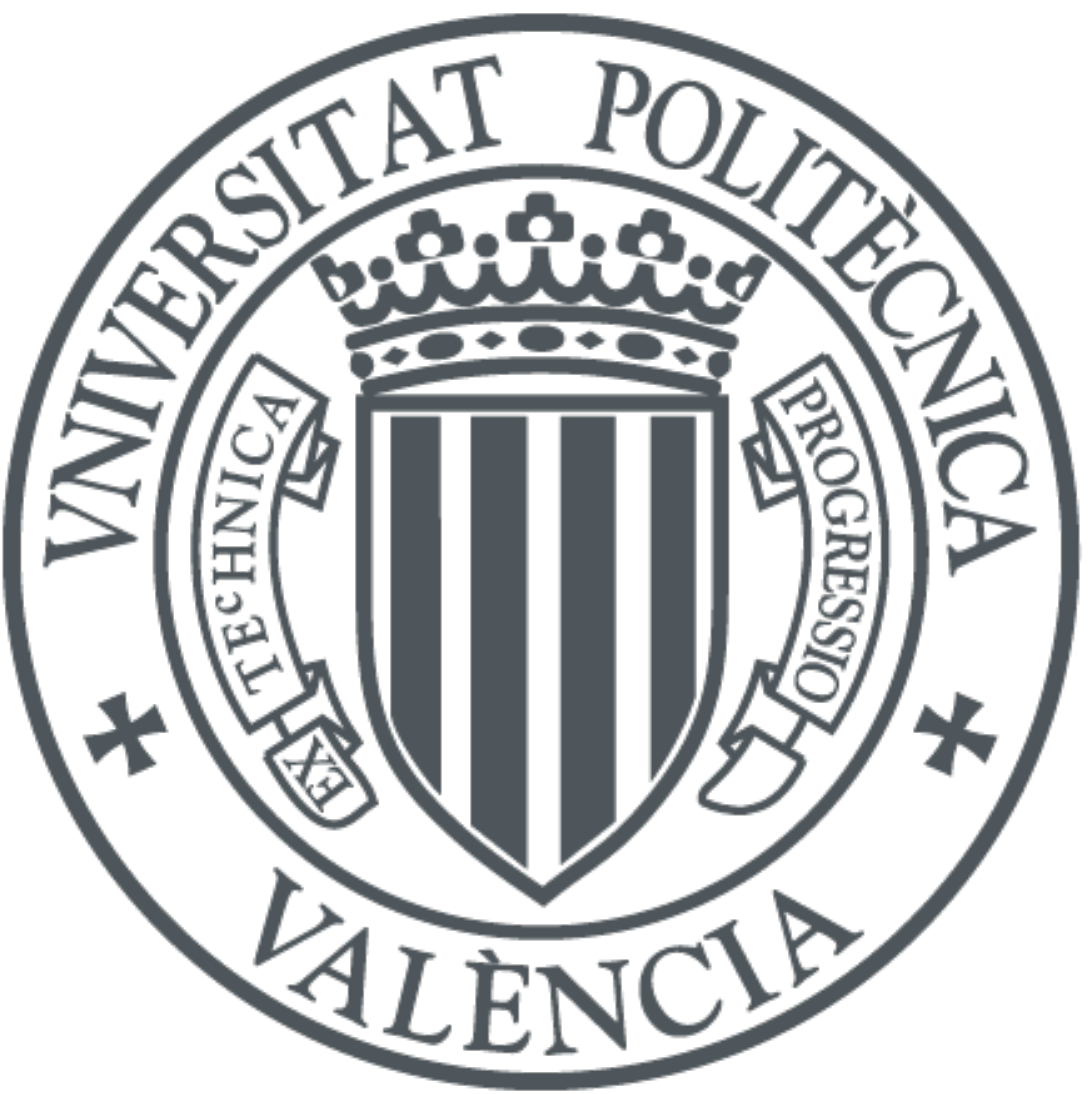

The final publication is available at

https://doi.org/10.1016/j.combustflame.2017.10.007

Copyright Elsevier

Additional Information 


\title{
Modal decomposition of the unsteady flow field in compression-ignited combustion chambers
}

\author{
A. J. Torregrosa ${ }^{\mathrm{a}}$, A. Broatch ${ }^{\mathrm{a}}$, J. García-Tíscar ${ }^{\mathrm{a}, *}$, J. Gomez-Soriano $^{\mathrm{a}}$ \\ ${ }^{a}$ CMT - Motores Térmicos, Universitat Politècnica de València, Camino de Vera, 46022 Valencia, Spain
}

\begin{abstract}
In this paper, the unsteady behaviour of a compression-ignited (CI) engine combustion chamber is studied by analyzing the results of a Computational Fluid Dynamics (CFD) model through the application of different flow decomposition techniques, aiming to resolve the underlying modal structure of the process. Experimental validation for the combustion simulation is provided, and a methodology for extracting coherent pressure information is proposed in order to provide a suitable input for different analysis methods. These range from straightforward Fourier transform techniques to more sophisticated modal decomposition approaches. In particular Proper Orthogonal Decomposition (POD) is shown to provide valuable insight into the time-spatial structure of the combustion flow field, allowing the establishment of correlations between pressure modes and physical parameters of the combustion, such as the injection timing or the chamber geometry. Dynamic Mode Decomposition (DMD) on the other hand is proven to successfully highlight the link between the frequency of the unsteady energy components and their spatial distribution within the chamber. Advantage is then taken of the modal characterization of the unsteady behaviour in the chamber to showcase how physical parameters such as the spray angle can be modified to optimize the acoustic signature of the combustion process, helping $\mathrm{CI}$ internal combustion engines reduce their acoustic environmental impact.
\end{abstract}

Keywords: DMD, POD, NVH, CFD, ICE, Noise

\section{Introduction}

Having served for more than a century as the primary means of powering transportation in industrialised societies, the reciprocating internal combustion engine is nowadays facing ever-increasing competition in the automotive market from another old [1] competitor, the electric motor.

While the main reason leading to regulatory pressure on the combustion engine is that of harmful combustion emissions, different solutions can be found to counter this issue, such as the use of catalytic converters, particle traps, advanced fuels and intelligent combustion strategies.

As oil, gas and coal-fired power plants are still notably featured in the generation mix of several countries, it is conceivable that the effective environmental footprint of electric vehicles could be matched by their thermal counterparts, at least until cleaner energy sources become widespread.

There is another aspect however in which electric cars enjoy a clear advantage over combustion-powered ones, that of NVH (Noise, Vibration and Harshness). Combustion implies, by definition, a sudden energy release which is in turn inherently noisy: energy is not only transferred to the piston; it also causes an unsteady flow field that resonates in the chamber and propagates through the engine block into the cabin and the environment [2].

*Corresponding author. Tel.: +34 963877 650, email: jorgarti@mot.upv.es
Although the combustion chamber is not the sole source of noise in passenger cars, since other sources such as turbochargers, pumps, wheels or aerodynamics are also relevant, it is certainly the most relevant one at low speeds and, thus, in urban environment where some authorities are already considering a permanent ban on thermal vehicles.

Therefore, it is essential to not only optimize combustion chambers of passenger cars to reduce the formation of pollutants, but to also consider carefully how the reduction of acoustic emissions may be achieved in the design process [3].

One of the most useful tools in the pursuit of optimized designs is Computer Fluid Dynamics (CFD), as a huge number of parameters can be tested without the need of costly and time-consuming experimental testing [4]. Once that a CFD solution has been validated, multiple changes can be simultaneously applied to the setup and solved in parallel, following strategies such as design of experiments [5], genetic algorithms [6, 7] and machine learning that autonomously refine a solution until an optimum is found.

However, to apply these algorithms and accomplish better designs of combustion chambers that not only address chemical pollutants but also the issue of combustion noise [8, 9], it is necessary to perform a detailed analysis of the CFD results, that allows the characterization of the unsteady behaviour of flow field in the chamber $[10,11]$.

In this paper, we apply different postprocessing techniques to a CFD model of a CI combustion chamber whose overall results in terms of pressure are also experimentally validated. We show how these techniques can reveal a better understanding of the combustion unsteady behaviour, reducing the need 
for guesswork and manual CFD postprocessing, alleviating the workload of design engineers and offering actionable information that can translate into quieter and more competitive combustion engines.

\section{Background}

In order to provide context for the problem being addressed in this work, a brief introduction of the state of the art in combustion noise issues produced by $\mathrm{CI}$ engines is provided in this section, along with the formulation of different analysis techniques of interest to better understand this problem.

\subsection{Resonant combustion noise of $\mathrm{Cl}$ engines}

Already since the work of Draper in 1938 [12], knock effects of SI combustion engines were investigated by comparison with the ideal vibration modes of a cylinder. One of the first investigations into noise production by CI engines was presented by Priede [13] who noted that "the rapid pressure rise caused by the sudden combustion of an appreciable fraction of the fuel-air mixture initiated also pronounced gas oscillations in the combustion chamber, and that these oscillations produce a broad peak on the cylinder-pressure spectrum and thus enhance the emitted noise in that frequency range".

He also indicated that "the frequency of gas oscillations is determined by the geometric shape of the combustion chamber", a concept that would be linked to resonance modes in a followup paper [14], in which he mentions the simple side-to-side mode already detected by Draper when sudden knocking events occurred in SI engines. However, neither had the means to resolve the actual, 3D gas oscillation modes caused by the combustion and the chamber geometry.

Targeting open chambers and assuming several simplifications, Hickling et al. [15] tried to improve upon purely theoretical formulas using a Finite Element Model (FEM). Still, his 16-element, quiescent gas FEM model lacked the capability to resolve actual flow or combustion, or even smallscale gas oscillations and thus, only a slight variation from the theoretical modal coefficients was obtained.

The potential of CFD for analyzing combustion chamber resonance modes was first demonstrated by Torregrosa, Broatch et al. $[8,10]$. However, although wave motion across the chamber was successfully resolved and compared with theoretical cylinder modes, no realistic initial flow field or combustion model were used, introducing instead small initial regions of high temperature and pressure simulating the ignition points.

Both shortcomings are overcome in the present investigation where three whole engine cycles are simulated, including the scavenging process, piston movement and valve movement profile. A realistic flow field including the swirling air motion is thus obtained in which the injection and combustion processes are modelled, accurately reproducing the pressure oscillations that are present in the real engine and are responsible for resonant combustion noise.
This numerical pressure data of the whole system, once validated, can then be analyzed through different techniques to reveal the real combustion modes directly, without the need of relaying on comparison or correlation with idealized analytic vibration modes of simple cylinders or cavities.

\subsection{Numerical identification of resonant modes}

A way to explore the spatial distribution of the acoustic flow field for different frequency-related phenomena of interest is to perform the Fourier transform at each cell record in the considered domain. Then, the amplitude of the transformed signal at the frequency of interest is used to color each cell, be it directly or through a smoothing interpolation.

This technique can be exploited in an attempt to identify the aforementioned actual modes of the combustion unsteady field. The spatial representation of selected Fourier traces that are manually selected based on inspection of the spectra can give a hint at the influence of the chamber geometry on the pressure oscillation patterns.

However, while this Fourier analysis method allows the obtaining of interesting results in a simple, straightforward way which moreover can be found already implemented in some commercial CFD codes, it is not without disadvantages.

Among them is the need to manually select the frequencies of interest, thereby having the risk of missing relevant frequencies, which are not known beforehand, and representing an increase of the workload of the design engineers. Time evolution of these spatial features is also lost unless Shorttime fast Fourier transform (STFFT) is attempted, at the cost of losing frequency resolution.

Also, in more complex geometries or flow structures such as those of turbulent jet ignition chambers, it could be difficult to exactly pinpoint relevant features, thereby requiring specific and intricate visualisation methods to reconstruct these modal structures.

\subsection{Proper Orthogonal Decomposition}

In order to address these limitations, more sophisticated analysis can be carried out to obtain the modal decomposition of the unsteady flow field. One of the most used among these is probably the Proper Orthogonal Decomposition (POD), also called Principal Component analysis (PCA) or Karhunen-Loève expansion [16], which originated in the field of probability theory and was first applied to the analysis of turbulent flow in 1967 [17]. As (rather poetically) put by Aubry in the aptly named paper "On the Hidden Beauty of the Proper Orthogonal Decomposition" [18], the objective of this technique is that:

"The flow is decomposed into both spatial and temporal orthogonal modes which are coupled: each space component is associated with a time component partner. The latter is the time evolution of the former and the former is the spatial configuration of the latter." 
In this way, this decomposition allows the identification of which spatial structures comprise the most energy of the flow field, which is understood as the superposition of all modes. The ordering of the contribution of each mode however, allows in principle a simplified yet meaningful reconstruction of the flow field.

Generally, information on the flow field evolution coming from either numerical simulations or experimental measurements ${ }^{1}$ will be naturally presented in a sequence of $N$ vectors $\mathbf{v}_{i}$ representing temporal snapshots that can be gathered in a matrix V:

$$
\mathbf{V}_{1}^{N}=\left\{\mathbf{v}_{1}, \mathbf{v}_{2}, \ldots, \mathbf{v}_{N}\right\}
$$

Each snapshot contains a quantity $M$ of scalar flow magnitudes which in the case of experimental results usually represent the velocity information. In the case of numerical simulations these snapshots may represent other useful information such as pressure, species, etc. These snapshots should be separated by a constant time step $\Delta t$.

POD can then be realized by diagonalizing the time-averaged spatial correlation matrix $\mathbf{V}^{\mathrm{T}} \mathbf{V}[19,20]$, by solving its associated eigensystem. However, $\mathbf{V}^{\mathrm{T}} \mathbf{V}$ can be very large, so an alternative approach is generally preferred, based on the Singular Value Decomposition (SVD) of V: [21, 22]

$$
\mathbf{V}=\mathbf{U} \boldsymbol{\Sigma} \mathbf{W}^{\mathrm{T}}
$$

In this way, $\mathrm{U}$ is an $M \times N$ matrix $^{2}$ whose columns contain the so-called POD spatial modes $\boldsymbol{\Psi}_{\mathbf{i}}$ (or left-singular vectors) that form an orthonormal basis of $\mathbf{V}$. These are also the eigenvectors of $\mathbf{V}^{\mathrm{T}} \mathbf{V}$, hence the validity of this alternate approach.

Next, $\boldsymbol{\Sigma}$ is an $M \times N$ diagonal matrix whose non-zero elements are the principal values $\sigma_{i}$ of $\mathbf{V}$, which correspond to the squared eigenvalues of $\mathbf{V}^{\mathrm{T}} \mathbf{V}$. They represent the contribution of each mode $\boldsymbol{\Psi}_{i}$ to the total energy of matrix $\mathbf{V}$, defined by Nikiforov [23] as $\sum \sigma_{i}$. Thereby, they indicate the relevance of each orthonormal mode in the total flow field.

Finally, $\mathbf{W}$ contains the right-singular vectors of $\mathbf{V}$, so therefore the rows of $\Sigma \mathbf{W}^{\mathrm{T}}$ describe the temporal evolution $\mathbf{a}_{i}(t)$ of the contribution of each spatial mode $\boldsymbol{\Psi}_{i}$, and thus the flow field can be described as a linear superposition of spatial and temporal information:

$$
\mathbf{V}(\mathbf{x}, t)=\sum_{i=1}^{N} \boldsymbol{\Psi}_{i}(\mathbf{x}) \mathbf{a}_{i}(t)
$$

The main advantage of the POD method is its capacity for dimensionality reduction. If the total flow field is reconstructed following Eq. 3 but with a reduced $L<N$ number of modes, the reconstructed flow field $\widetilde{\mathbf{V}}$ is the closest one to the original, in the sense that the Frobenius norm of the difference between the original and the reduced field is the minimum achievable for a reduced $\widetilde{\mathbf{V}}$ of rank $L$.

\footnotetext{
${ }^{1}$ For instance, from experimental Particle Image Velocimetry (PIV) measurements that capture a sequence of velocity field snapshots.

${ }^{2}$ If the "economy" version of the SVD algorithm is used in order to optimize computation time.
}

This can be proved by realizing that the partial summatory reconstruction is equivalent to forming a reduced $\widetilde{\Sigma}$ with $\sigma_{i}=0$ when $L<i<N$ and then obtaining $\widetilde{\mathbf{V}}=\mathbf{U} \widetilde{\Sigma} \mathbf{W}^{\mathrm{T}}$. The Eckart-Young theorem [24] ensures that a matrix of rank $L$ reconstructed in this way minimizes $\|\mathbf{V}-\widetilde{\mathbf{V}}\|_{F}$.

For many applications, especially in the field of computational fluid dynamics where simulations feature very large matrices, dimensionality reduction is crucial in order to facilitate further use of the results such as vibroacoustic propagation, structural mechanics, aeroelasticity simulations, or preparing inlet conditions for Large Eddy Simulations [25, 26].

Principal drawbacks of this method include that in some cases energy may not be the most relevant metric in characterizing the relevance of flow structures [19], and that the use of second-order flow statistics such as the time-averaged spatial correlation tensor can result in a loss of information such as phase [18], failing to capture the dynamics of the coherent structures of the flow [27].

In cases where the flow experiments highly transient regimes, it is thus possible that short-lived flow modes with small contribution to the total energy of the system may be missed from the POD energy ranking [19]. Moreover, while modes resulting from POD exhibit spatial orthogonality, they can contain multi-frequency temporal content [28, 29]. This means that a particular flow structure can be excited by different phenomena and at different frequencies, yet remain spatially coherent in time and thus result in a higher energy POD mode. In the following subsection, a more recent modal decomposition technique specifically introduced [19] to address both of these issues will be described.

Linking the unsteady spatial structures with a specific phenomena requires, as a consequence of POD shortcomings, some guesswork based on prior knowledge of the excitation mechanisms. A good example of this is the application of POD to the CFD simulation of an engine manifold presented by Sakowitz et al. [29], where it can be seen how the first POD mode contains different frequency content originated from both the engine firing and the valve opening. However, the combustion chamber itself is not included in their work.

Other authors have applied POD to combustion processes, be it through CFD simulations [30], or experimentally obtaining the flow snapshots with the aid of optical techniques such as PIV [31, 32, 33, 34], chemiluminiscence [35] or raw luminosity [36], but most of these results are restricted to continuous combustion cases such as those of gas turbines or industrial burners, focusing on the thermoacoustics originated by the open flame oscillations and not on the more explosive, confined acoustics of reciprocating ICE combustion chambers.

Although some authors have specifically addressed ICE combustion issues through POD, these studies have been focused on cycle-to-cycle variation analysis [36, 37, 38], sparkignition misfires [39] or the evolution of a particular species [40], whereas the pressure resonance issues of CI combustion chambers addressed in this work has not been yet investigated using this method. 


\subsection{Dynamic Mode Decomposition}

An alternative decomposition approach known as Dynamic Mode Decomposition (DMD) exists where instead of an optimal orthogonal basis, coherent flow phenomena at each frequency are sought. A relatively recent technique for modal flow decomposition [41, 19, 42, 28], its application to combustion problems is still scarce, and focused on the analysis of experimental PIV data $[43,44]$ such as velocity and vorticity fields in combustors.

DMD is realized by estimating the eigenmodes and eigenvectors of the linear but infinite-dimensional Koopman operator of a given system, which describes its nonlinear, finitedimensional dynamics $[42,45]$. In contrast with POD, DMD eigenvalues represent a single frequency, along with the rates of decay and growth of the spatial DMD modes, hence allowing the identification of coherent but weakly-energetic modes in highly transient regimes [46].

Evaluation of the relevance of each mode to the dynamics of the system is usually performed by computing the norm of the mode, although Dahan et al. reported [47] that in some cases it may be preferable to order the modes attending to their frequency rather that their norm, as some modes may feature a growth rate of less than the unity, and thus they may be damped over time.

Furthermore, another advantage of the DMD and POD techniques is that it can be proved $[19,27]$ that processing only subdomain data is possible, be it this subdomain a small region of the domain or even a projection of the $3 \mathrm{D}$ field into a $2 \mathrm{D}$ plane, as no spatial arrangement is assumed by either method. This is convenient as post-processing can be restricted to an area of the flow where relevant phenomena are expected, saving computational effort.

As several variations for implementing a DMD strategy already exist in the literature only the basic concept originally proposed by Schmid [19] will be shown in this section. We recall the matrix $\mathbf{V}_{1}^{N}$ introduced in subsection 2.3 that contains $N$ snapshots of the flow field. These are assumed to be linearly related through a certain matrix $\mathbf{A}$, which is assumed to remain approximately constant during the time $N \Delta t$ spanned by the sequence:

$$
\mathbf{v}_{i+1}=\mathbf{A} \mathbf{v}_{i}
$$

Even if the flow field is non-linear, matrix A will provide a linear tangent approximation of the dynamic characteristics of the flow. The objective is now to extract its eigenvalues and eigenvectors which, will be the DMD eigenvalues and the DMD modes of the flow. Combining both expressions in matrix form we can write that:

$$
\mathbf{V}_{2}^{N}=\mathbf{A V}_{1}^{N-1}
$$

Where $\mathbf{V}_{2}^{N}$ contains snapshots 2 to $N$ and $\mathbf{V}_{1}^{N-1}$ contains snapshots 1 to $N-1$. As $M$ for CFD simulations is usually in the order of $10^{6}$ and $N$ must comprise a sensible amount of time steps in order to ensure that the dynamic characteristics are well captured, directly performing the eigendecomposition of $\mathbf{A}$ is usually too computationally expensive.
In order to overcome this problem different strategies exist, such as reformulating 5 into a companion matrix that can be solved through a QR decomposition [27, 41, 20]. A more numerically stable method however is to make use again of the Singular Value Decomposition (SVD) [19, 28, 29, 42] to obtain $\mathbf{V}_{1}^{N-1}=\mathbf{U} \Sigma \mathbf{W}^{\mathrm{T}}$. Then, equation 5 becomes:

$$
\mathbf{V}_{2}^{N}=\mathbf{A} \mathbf{\Sigma} \mathbf{W}^{\mathrm{T}}
$$

From this expression it is now possible to manipulate the terms that are already known in order to define a new matrix $\widetilde{\mathbf{S}}$, which will be thus related to A in the following way:

$$
\widetilde{\mathbf{S}} \triangleq \mathbf{U}^{\mathrm{T}} \mathbf{V}_{2}^{N} \mathbf{W} \boldsymbol{\Sigma}^{-1}=\mathbf{U}^{\mathrm{T}} \mathbf{A U}
$$

It can be seen that this relationship between $\widetilde{\mathbf{S}}$ and $\mathbf{A}$ matches the definition of matrix similarity. Thus, $\widetilde{\mathbf{S}}$ is similar to $\mathbf{A}$ and as a consequence their eigenvalues $\lambda_{i}$ are the same, with the advantage that $\widetilde{\mathbf{S}}$ is of reduced size and easier to solve. The DMD modes $\Phi_{i}$ can finally be calculated from the eigenvectors $y_{i}$ of $\widetilde{\mathbf{S}}$ as:

$$
\Phi_{i}=\mathrm{U}_{i}
$$

The frequency associated with each spatial mode can be recovered by evaluating the imaginary part of each complex eigenvalue, and taking into account the time step $\Delta t$ between snapshots:

$$
f_{i}=\frac{\omega_{i}}{2 \pi}=\frac{\mathfrak{I}\left\{\ln \left(\lambda_{i}\right)\right\}}{2 \pi \Delta t}
$$

As for ranking the contribution of each mode to the total flow field, several approaches can be followed. Since many eigendecomposition routines normalize the eigenvectors [48], simply computing their $\ell_{2}$ norm is not always reliable.

The energy of the modes can be recovered by solving a linear system in which the DMD-reconstructed field multiplied by unknown amplitudes is compared against a selected snapshot [48]. It is also possible to optimize these amplitudes to promote a more sparse reconstruction of the field with fewer modes [28].

Another ranking metric was considered by Schmid [19] in his original DMD formulation, based on the computation of the modes' coherence in time. This can be done by first calculating matrix G [49]:

$$
\mathbf{G}=\mathbf{V}_{1}^{N-1} \Sigma^{-1} \mathbf{Y}
$$

Where $\mathbf{Y}$ is the matrix that gathers the eigenmodes $y_{i}$ of $\widetilde{\mathbf{S}}$. Then for each column $\mathbf{g}_{i}$ of $\mathbf{G}$, the coherence $E_{i}$ of each DMD mode $\Phi_{i}$ can be obtained as the inverse of its norm:

$$
E_{i}=\left\|\mathbf{g}_{i}\right\|^{-1}
$$

A disadvantage of this solution for ranking the modes is that the true magnitudes of the vectors for flow reconstruction purposes remain unknown. If flow reconstruction is desired, for instance aiming to simplify the application of the Ffowcs Williams-Hawkings acoustical propagation procedure [50] as done by Dahan et al. [47], solving the amplitudes in a reference snapshot as done by the dymode code [48] or the use of the sparsity-promoting procedure by Jovanović et al. [28] are recommended instead. 


\section{Numerical model}

Since an inherent goal of this research is to contribute to combustion noise fundamental knowledge, the modelled engine was conscientiously selected for dealing with current design tendencies of the automotive industry for compressionignited engines. The selected engine is a downsized high speed direct injection (HSDI) engine equipped with a common rail system and a turbocharger. The geometry of the pipes and combustion chamber used in this work and shown in Fig. 1 was created by reverse-engineering the actual parts.

The numerical model of one cylinder of the engine, including intake and exhaust pipes, was build in the commercial CFD platform CONVERGE. The three-dimensional domain was discretized in structured mesh of hexahedrons with a cell size of $3 \mathrm{~mm}$. To ensure an accurate prediction of the flow properties, the mesh size was reduced in areas where physical and chemical phenomena increase their complexity by applying a grid scale factor. This parameter changes the base grid size according to:

$$
L_{\text {scaled }}=\frac{L_{\text {base }}}{2^{r}}
$$

where $r$ is the scale factor and, $L_{\text {base }}$ and $L_{\text {scaled }}$ are the base cell size and the new grid size, respectively.

A scale factor of three was applied to the combustion chamber and ports walls, in the spray zones and in the whole combustion chamber during the combustion process. These adjustments improve the thermal boundary layer prediction, the reflection and the interaction of the pressure waves and the precision in the chemical reactions and spray modelling processes (atomisation, breakup, coalescence, etc.).

Additionally, the code used an adaptive mesh refinement algorithm (AMR) to increase the spatial resolution where both velocity and temperature gradients are significant. The total number of cells depended on the simulation timing and varied among $1.5 \times 10^{6}$ cells at the bottom dead center and $0.5 \times 10^{6}$ cells at the top dead center.

The simulation encompasses a complete engine cycle between two consecutive exhaust valve opening (EVO) timings. The gas exchange process (GEP) simulation was only performed to provide more realistic flow conditions for the combustion phase calculations. Hence, the solutions obtained during the GEP were not considered for the following studies. The time step is optimized by a dynamic strategy based on two distinct Courant number definitions.

This method estimates the time step at every calculation step from the maximum local velocity registered at the smallest cells of the domain. The velocity of the flow was taken as limiting factor during the compression and injection phases, whereas the speed of sound was considered during the combustion. As a result, the computational time was reduced without loss of accuracy regarding the simulation of the wave oscillations and reflections that are produced by the combustion heat release, and are crucial in this investigation.

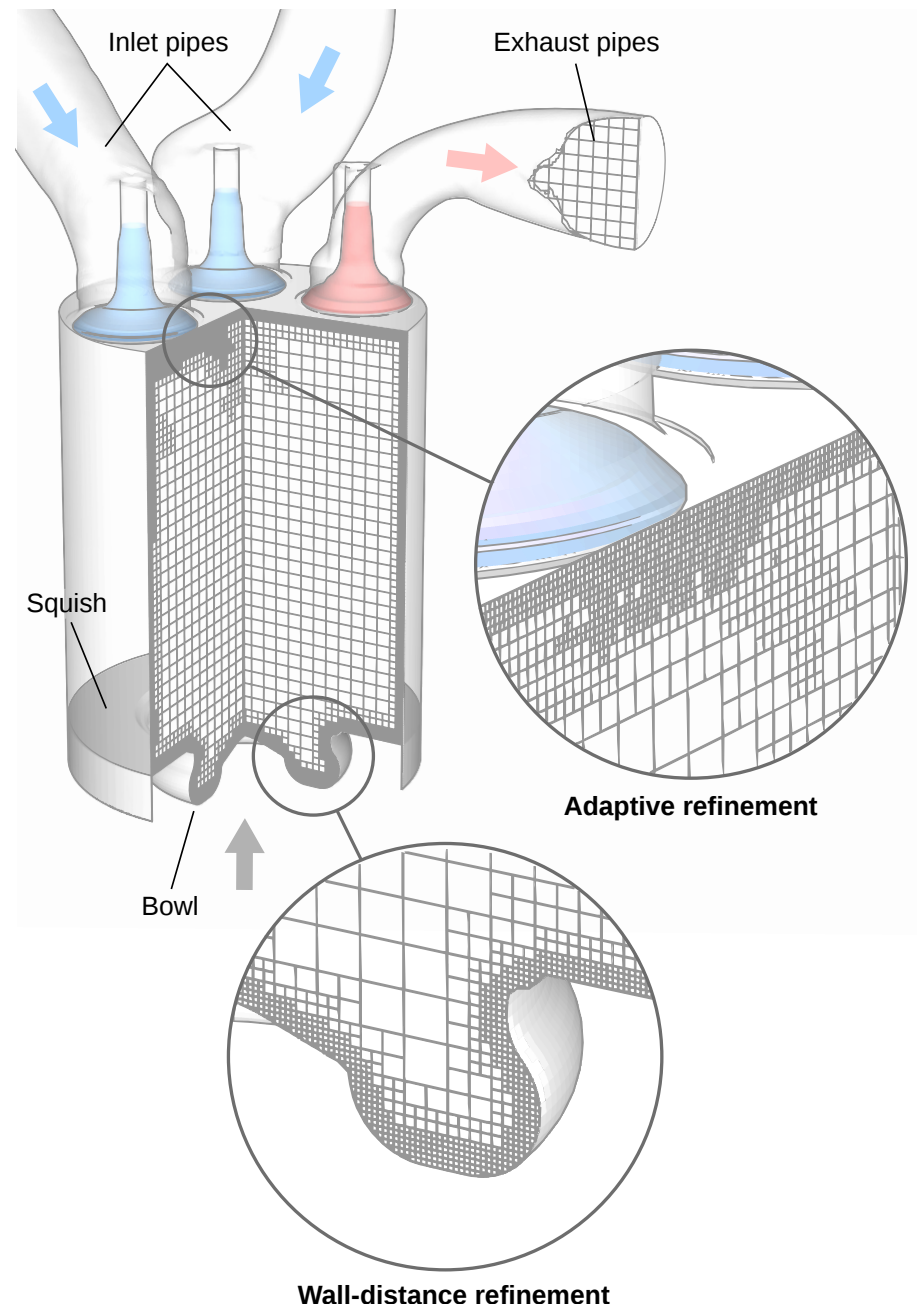

Figure 1: Computational domain at IVC, including the intake and exhaust pipes and valves, cylinder walls and the combustion chamber on top of the piston. Details of the mesh sizing and its refinements at different zones are also provided.

Mass, momentum and energy conservation equations were numerically solved with the finite volume method and a second-order accurate spatial scheme. The species transport were calculated by the mass fraction governing equations of all species in the domain.

Turbulent flow structures were approximated by the renormalization group (RNG) $k-\varepsilon$ model [51] coupled with a heat transfer model [52] for estimating the wall heat fluxes. The Redlich-Kwong approach [53] was selected as equation of state required for calculating the compressible flow properties. Finally, a modified Pressure Implicit with Splitting of Operators (PISO) method [54] was used for solving the pressure-velocity coupling.

This approach has been successfully used in many numerical simulations of compression-ignited combustion presented in the literature, $[55,56,57]$. Coupled with appropriate combustion models, Wright et al. [58] demonstrated that this turbulence model allows accurate reproduction of autoigni- 
tion, while Han and Reitz [59] established that realistic rates of heat release can be achieved, both conditions of capital importance when addressing, as in this work, the unsteady oscillations produced by the combustion.

Fuel injection was modelled by the standard Discrete Droplet Model (DDM) [60] while the spray properties such as liquid atomisation and drop breakup were simulated by using the Kelvin-Helmholtz Rayleigh-Taylor (KH-RT) model [61]. The physical properties of the fuel were predicted by choosing the n-heptane as a surrogate.

Concerning combustion, the code approaches the detailed chemical kinetics through a direct integration of the surrogate fuel chemical mechanism by means of the SAGE (detailed transient chemistry solver) solver [62]. The chemical mechanism was based on a Primary Reference Fuel (PRF) blend of n-heptane and iso-octane, deactivating the iso-octane reactions so as to predict the Diesel ignition features. An adapted reaction mechanism was derived from the ECR-Multichem mechanism [63] for PRF oxidation. this mechanism characterises the Diesel fuel chemistry with 42 species and 168 reactions.

Boundary conditions have been set by simplified models and also with direct measurements. Wall temperatures of the cylinder liner, piston, head, intake-exhaust ports and valves were considered isothermal and estimated by a lumped model [64]. The inflow and outflow boundaries located at the end of the intake-exhaust ports were established as the mean value of the measurements of instantaneous pressure and temperature.

Initial conditions of the flow (pressure, temperature, velocity field and species concentration) were determined by a combination of OD modelling [65] and direct measurements. Then, three full engine cycles were calculated, as the first simulated cycle is influenced by these simplistic initial conditions. The $3^{\text {rd }}$ cycle however was proved to be correctly converged to realistic conditions.

On the engine operation map, the region framed in the low-medium speed and low-medium load is traditionally accepted as the most critical operation range from the point of view of noise emissions. A particular operation condition was therefore chosen, at medium speed (2400 rpm) and medium load (168 Nm), thus being representative of this specific operation range.

\subsection{Model validation}

In order to ensure that representative pressure data was captured by the simulation, an experimental validation campaign was carried out at the facilities of CMT-Motores Térmicos, where pressure data from the same engine that was used in the reverse engineering was recorded for 50 cycles.

The guidelines introduced by Broatch et al. [66] were used as a reference for the validation procedure. The pressure trace measured at a specific point inside the combustion chamber was contrasted to that computed by the CFD model at exactly the same location of the transducer.
Overall Noise (ON) [67] was computed for all experimental cycles, resulting in a mean value of $89.96 \mathrm{~dB}$ with a standard deviation of $1.47 \mathrm{~dB}$. The $3^{\text {rd }}$ simulated cycle presented an $\mathrm{ON}$ value of $90.96 \mathrm{~dB}$, this is, within the aforementioned experimental deviation. For instance, one of the experimental cycle was rated at $\mathrm{ON}=91.12 \mathrm{~dB}$, this is, a difference of only $0.16 \mathrm{~dB}$ against from numerical $3^{\text {rd }}$ cycle.

In Fig. 2, the in-cylinder pressure data from the selected experimental cycle, including the experimental standard deviation data, is plotted against the $3^{\text {rd }}$ simulated cycle in both time and frequency domains. It can be seen in the zoomed view how the resonant oscillation process studied in this paper is self-similar from cycle to cycle: the cyclical dispersion is very small and the same oscillation peaks can be consistently identified.

On the bottom plot, pressure spectral density is shown, indicating the source mechanism of each part of the spectral signature, following the method presented by Strahle [68] where the mechanical compression and mean combustion pressure trace are subtracted to identify the cut-off frequency (in this case $\sim 4 \mathrm{kHz}$ ) above which the unsteady pressure oscillations are preponderant
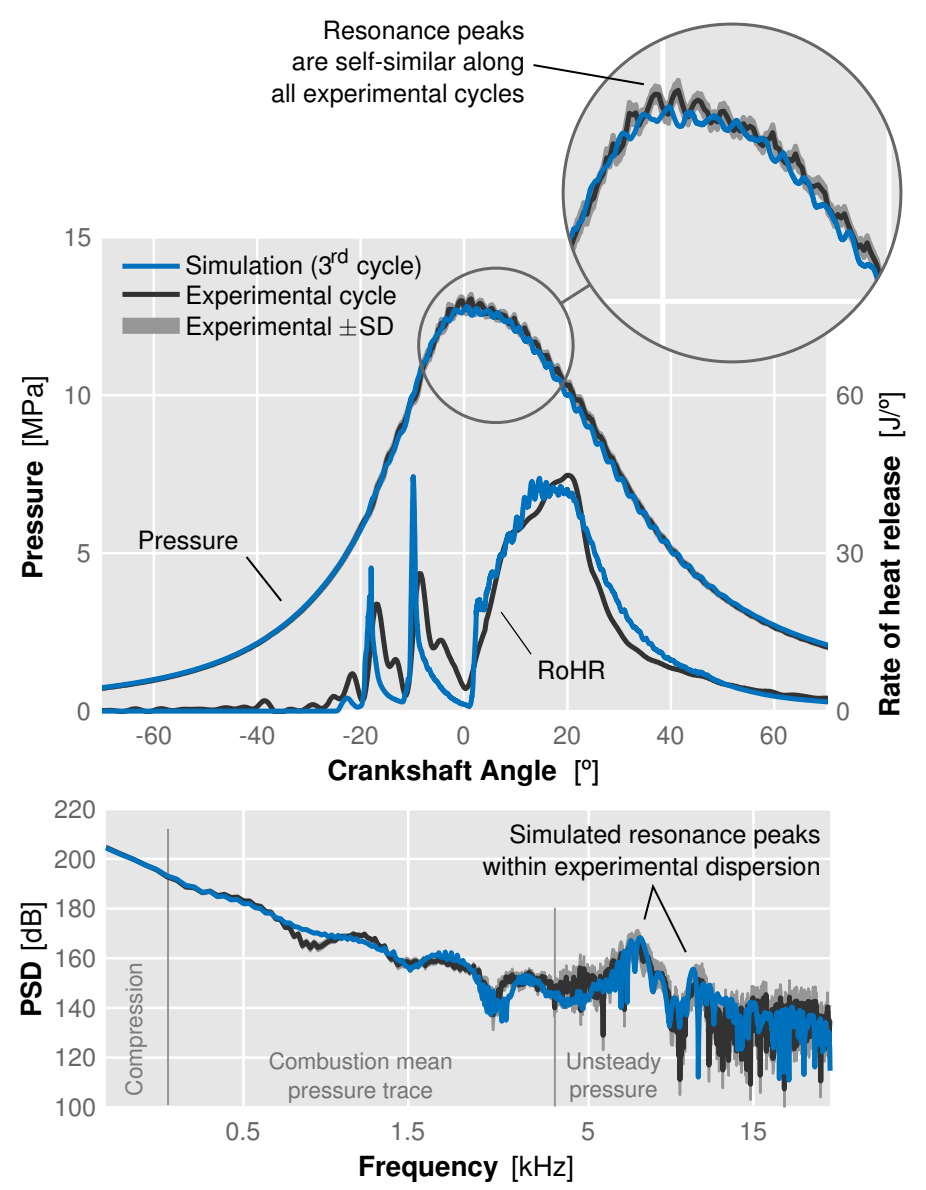

Figure 2: Experimental validation of the numerical model in terms of in-cylinder pressure and rate of heat release (top) and frequency content of the pressure signal (bottom). 
It can be seen in the plot that the simulated data in this last part of the spectrum that constitutes our range of interest is completely within the experimental standard deviation, thus validating previous research suggesting that CI combustiongenerated wave interaction is well captured by PISO-solved RNG $k-\varepsilon$ numerical schemes.

Lastly, in addition to the in-cylinder pressure comparison, the rate of heat release (RoHR) is also included in Fig. 2 (top) for deeper validation of the combustion process. The experimental RoHR is obtained by solving the energy equation by direct measurements and assuming several simplifications [65]. Again, as reported by Han and Reitz [59], a good match between experimental and simulated RoHR data is obtained.

This fact, linked to the aforementioned low cycle-to-cycle dispersion of CI engines, contributes to visualize why the selected URANS scheme provides the accurate reproduction of experimental data shown in this section: even if the local fluctuation components of the Navier-Stokes equations are neglected, the macroscopic oscillations of the pressure waves due to the autoignition of the premixed fuel-air blend are still successfully reproduced.

In contrast, in spark-ignition (SI) engines the pressure oscillations are caused by the propagating flame front which is a process heavily dependent on an accurate resolution of the local turbulent features. Hence, in simulations of SI combustion ICEs more realistic turbulent models such as Large Eddy Simulation (LES) are typically used $[69,70,71]$, since the local fluctuating components are kept up to a certain scale.

\subsection{Data preparation}

Results from the simulation were exported by means of a custom UDF (user defined function). After each simulated time step converged, pressure data was saved to a text file, each row containing cell centroid coordinates $x, y$ and $z$ and corresponding pressure $p$.

However, it must be taken into account that modern CFD codes sometimes have the ability of automatically refining or coarsening the mesh as the simulation advances, in order to optimize solving time. Cells are also dynamically created and destroyed as the cylinder moves. As a consequence, cells are not necessarily preserved across snapshots.

It was thus necessary to perform a preliminary postprocessing step in order to obtain coherent pressure records. A reference snapshot was first chosen, and then a subset of $10^{5}$ random cells was selected from it in order to speed computations while still ensuring a good spatial resolution.

The rest of the snapshot files were then scanned to find the best match for the selected reference cells. Given each snapshot $i$, this best match for each reference cell $j$ was determined by calculating ${ }^{3}$ the squared Euclidean distance between the reference cell and the entire set of cells of the snapshot:

$$
\mathbf{d}_{i, j}^{2}=\left(\mathbf{X}_{i}-x_{j}^{\mathrm{ref}}\right)^{2}+\left(\mathbf{Y}_{i}-y_{j}^{\mathrm{ref}}\right)^{2}+\left(\mathbf{Z}_{i}-z_{j}^{\mathrm{ref}}\right)^{2}
$$

\footnotetext{
${ }^{3}$ The authors noticed that the proposed vector approach appeared to be at least an order of magnitude faster than MATLAB's default function dsearchn $(X, X I)$.
}

In the next step, $\min \left(\mathbf{d}_{i, j}^{2}\right)$ is evaluated. If $\min \left(\mathbf{d}_{i, j}^{2}\right)=0$, at position $k$, a cell exists in snapshot $i$ that exactly matches the reference cell $j$, and thus its pressure value $p_{k}$ is added to the global snapshot matrix, $\mathbf{V}(j, i)=p_{k}$.

However, it could happen that $\min \left(\mathbf{d}_{i, j}^{2}\right)>0$, this is, that no exact cell match is found for the previously mentioned reasons of mesh refinement or coarsening and of piston displacement. In order to differentiate between both issues, a maximum cell centroid displacement $d_{c, \max }$ due to refinement and coarsening can be calculated taking into account CONVERGE input parameters $L$ and $r$ :

$$
d_{c, \max }=\sqrt{3\left(\frac{L}{2}-\frac{L}{2^{r+1}}\right)^{2}}
$$

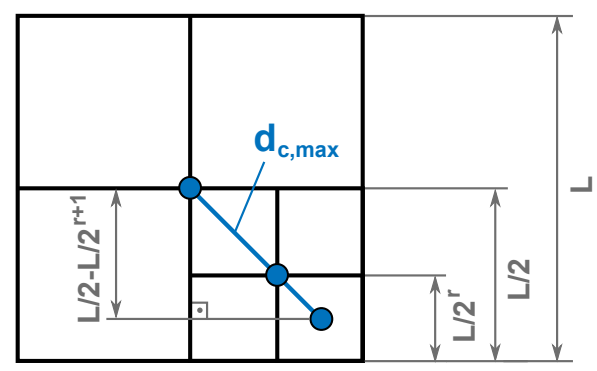

Figure 3: Simplified example of the maximum allowable centroid distance during the cell search process, for the case of $r=2$ in 2D. Extension to 3D cubic cells is straightforward.

The derivation of this expression is exemplified in Fig. 3. If $\min \left(\mathbf{d}_{i, j}^{2}\right) \leq d_{c, \text { max }}$, at position $k$, then cell $k$ is related to reference cell $j$ through a mesh refinement or coarsening process and its pressure value $p_{k}$ is again stored at $\mathbf{V}(j, i)$.

If however $\min \left(\mathbf{d}_{i, j}^{2}\right)>d_{c, \text { max }}$, it can be concluded that the position of reference cell $j$ has been blocked by the piston in snapshot $i$, and thus the pressure record at that location cannot be used. A NaN value is then stored in $\mathrm{V}(j, i)$.

Finally, rows of $\mathbf{V}$ containing NaNs are culled, thereby obtaining an snapshot matrix $\mathbf{V}$ of continuous pressure records at approximately constant spatial positions, suitable to apply the discussed postprocessing techniques. As CONVERGE may sometimes introduce NaNs as well, this step ensures that the data is valid for further postprocessing.

It should also be mentioned that if the simulation was carried out with a variable time step to optimize the computation speed, an additional interpolating step must be then performed in each row of $\mathbf{V}$ to obtain snapshots that are equally sampled in time.

\section{Postprocessing, results and discussion}

In this section, the postprocessing techniques outlined in section 2 are applied to the results of the combustion simulation, already prepared into an snapshot matrix $\mathbf{V}$ of coherent pressure information. 

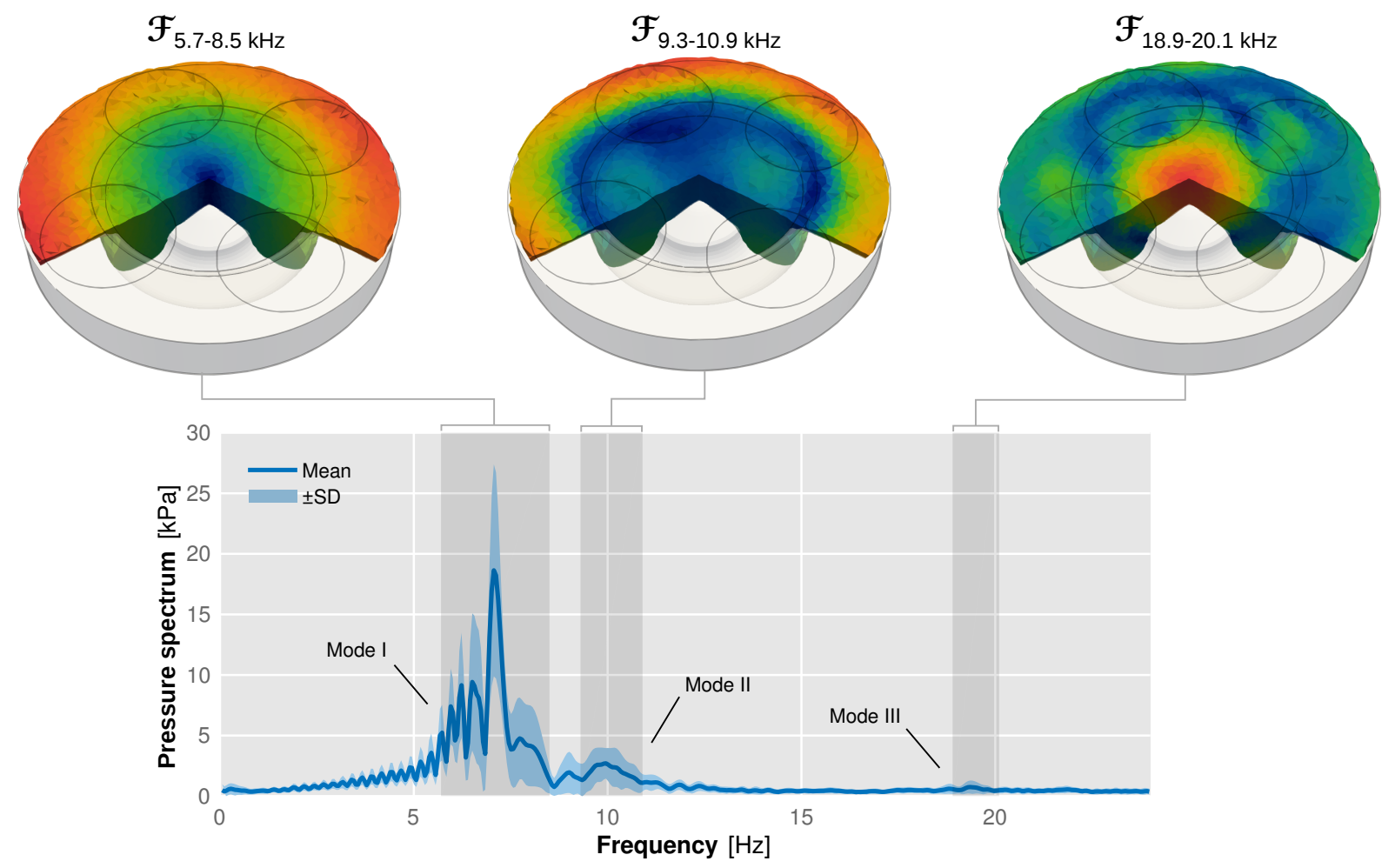

Figure 4: Fourier analysis of the combustion pressure signal. Pressure spectrum, averaged across all selected cells in the domain, is plotted along its standard deviation (SD). Three characteristic "bumps" are identified and marked, accompanied by the spatial distribution of the Fourier transform restricted to each selected frequency range.

\subsection{Fourier analysis}

With the aim of giving a first approach of the unsteady flow field responsible for acoustic emissions, a straightforward Fourier analysis was performed by following the procedure outlined in section 2. Resonant modes and their spatial distribution were identified through pressure spectra inspection.

The interest of the analysis is focused on the medium-high frequencies, in whose spatial modes particular pressure distributions and their acoustic contribution can play an important role. Therefore, the homogeneously-distributed, steady pressure evolution was subtracted from each cell pressure record in order to focus on the unsteady phenomena propagating in the chamber.

Once this procedure was applied to all rows of $\mathbf{V}$ matrix, the Fast Fourier Transform was applied to every resulting pressure signal at each considered cell. Results are presented in Fig. 4, in which pressure spectra were averaged across all selected cells in the domain and its standard deviation is displayed to denote the spatial variation of the pressure oscillations.

Three distinct "bumps" are noticed and their associated frequencies can be identified, similar to the work of Torregrosa et al. [8]. These allow to obtain the Fourier transform restricted to each cell and selected frequency band and, hence, the spatial distribution of the resonant modes can be visualized through the volume renders displayed in Fig. 4 (top).
Mode I, located between $5.7-8.5 \mathrm{kHz}$, is clearly the most relevant among all the identified ones. The reflection of the pressure waves in the transversal direction makes this mode essentially a transversal mode with a single node line. Therefore, the higher amplitudes are oppositely localised in the squish zone of the chamber.

The second and third resonant modes seem to be gathered at $9.3-10.9 \mathrm{kHz}$ and $18.9-20.1 \mathrm{kHz}$, respectively. Their amplitude is significantly lesser, especially in the case of mode III. The second resonant mode results from interaction among the oscillations which are excited in the squish and also inside the bowl. The higher amplitudes are established near the cylinder walls as in the mode I, but in this case, an additional source of oscillations is located within the bowl.

In contrast with the previous modes, the highest frequency mode oscillations are aligned according the symmetry of the combustion chamber, showing an annular node region, and its oscillation pattern seems to be focused inside the bowl.

\subsection{POD decomposition}

Proper Orthogonal Decomposition was then performed as described in section 2. Orthonormal POD modes $\boldsymbol{\Psi}_{i}$ and their corresponding principal values $\sigma_{i}$ were obtained, together with temporal evolution coefficients $\mathbf{a}_{i}$. 


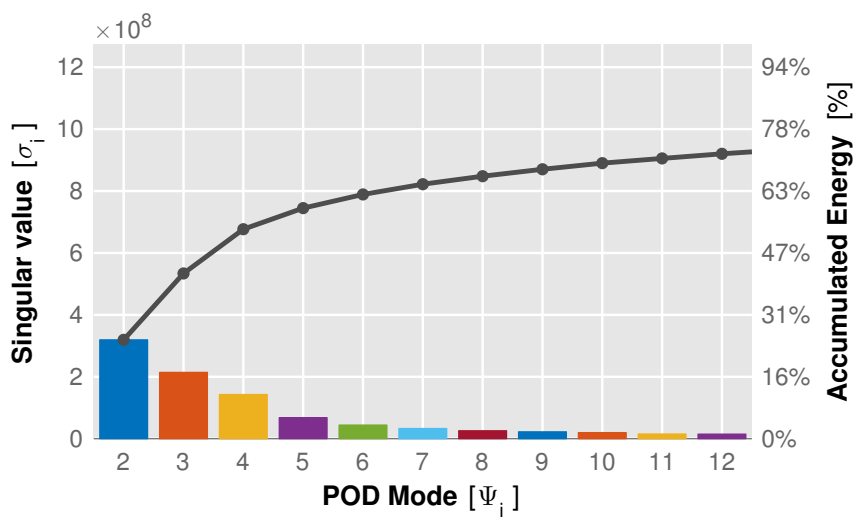

Figure 5: Pareto chart showing the singular values associated to POD modes $\Psi_{2-12}$ and the accumulated contribution to the remaining energy after $\Psi_{1}$ is disregarded (since it only contains the well-known information of the homogeneous mean pressure).

In order to characterize the relevance of each mode, their contributions to the total energy of the snapshot matrix are computed with the help of the principal values. Since the mean pressure evolution in the chamber is not subtracted, the first spatial mode $\boldsymbol{\Psi}_{1}$ is homogeneously distributed through the chamber, with its singular value $\sigma_{1}$ representing $97.5 \%$ of the matrix energy.

As in this paper the main interest lies in extracting the unsteady behaviour of the combustion itself, rather than that of the compression-expansion cycle, the first mode related to the mean homogeneous pressure can be disregarded. The remaining energy is thus distributed among the subsequent modes as shown in the Pareto chart of Fig. 5.

It can be seen in this figure that POD modes $\boldsymbol{\Psi}_{2-12}$ gather approximately $70 \%$ of the remaining energy, with $50 \%$ being gathered just by modes $\Psi_{2-5}$. Although not shown in the graph, $80 \%$ of the remaining energy is represented by modes $\boldsymbol{\Psi}_{2-27}$ and finally modes $\boldsymbol{\Psi}_{2-180}$ sum up to $99 \%$. The rest of the modes $\boldsymbol{\Psi}_{181-862}$ represent just 1\% of the remaining energy, demonstrating the usefulness of POD for dimensionality reduction.

Besides the scrutiny of the singular values to measure the overall energy contribution of each mode, the information contained within the time coefficients $\mathbf{a}_{i}=\sigma_{i} \mathbf{W}_{i}^{\mathrm{T}}$ allows the analysis of the evolution of each mode in the time and frequency domains.

In Fig. 6 the normalized time coefficients $\mathbf{a}_{i}(t)$ of POD modes $\boldsymbol{\Psi}_{1-12}$ are plotted for each time step of the CFD simulation. Mode $\boldsymbol{\Psi}_{1}$ has been independently scaled with $\max \left(\boldsymbol{\Psi}_{1}\right)$ to allow its representation along the rest, while each remaining mode $\boldsymbol{\Psi}_{2-12}$ has been scaled with $\max \left(\boldsymbol{\Psi}_{2-12}\right)$ in order to preserve the scaling between them due to their corresponding singular values $\sigma_{i}$.
It is clear from the figure that, while $\boldsymbol{\Psi}_{1}$ describes the smooth evolution of the mean pressure in the chamber, the rest of the modes describe the acoustic instabilities associated to the combustion process. This is made more evident by Fig. 7 where the frequency content associated to each mode is presented.

Inspecting the time evolution of the POD modes shown in Fig. 6, clear relationships can be found between the inception of the modes and the timing of the combustion of the different injections of the cycle, denoted in the figure by $C_{1-3}$. These instants have been found by calculating the time step at which $10 \%$ of the fuel of each injection is consumed.

From the zoomed-in detail view included in the figure, it can be easily seen how the onset of modes $\boldsymbol{\Psi}_{2,3,5}$ is coincident with the time of $C_{1}$, although the amplitude rise is much more pronounced in the dominant mode $\boldsymbol{\Psi}_{2}$ while in mode $\boldsymbol{\Psi}_{3}$ the time evolution is much more progressive. On the other hand, mode $\Psi_{4}$ shows little relevance after $C_{1}$, only exhibiting a sudden rise in amplitude at the precise time of $C_{2}$. None of these first 12 modes seems to be triggered by $C_{3}$, confirming the preponderance of $C_{1}$ and $C_{2}$ in creating the resonant field.

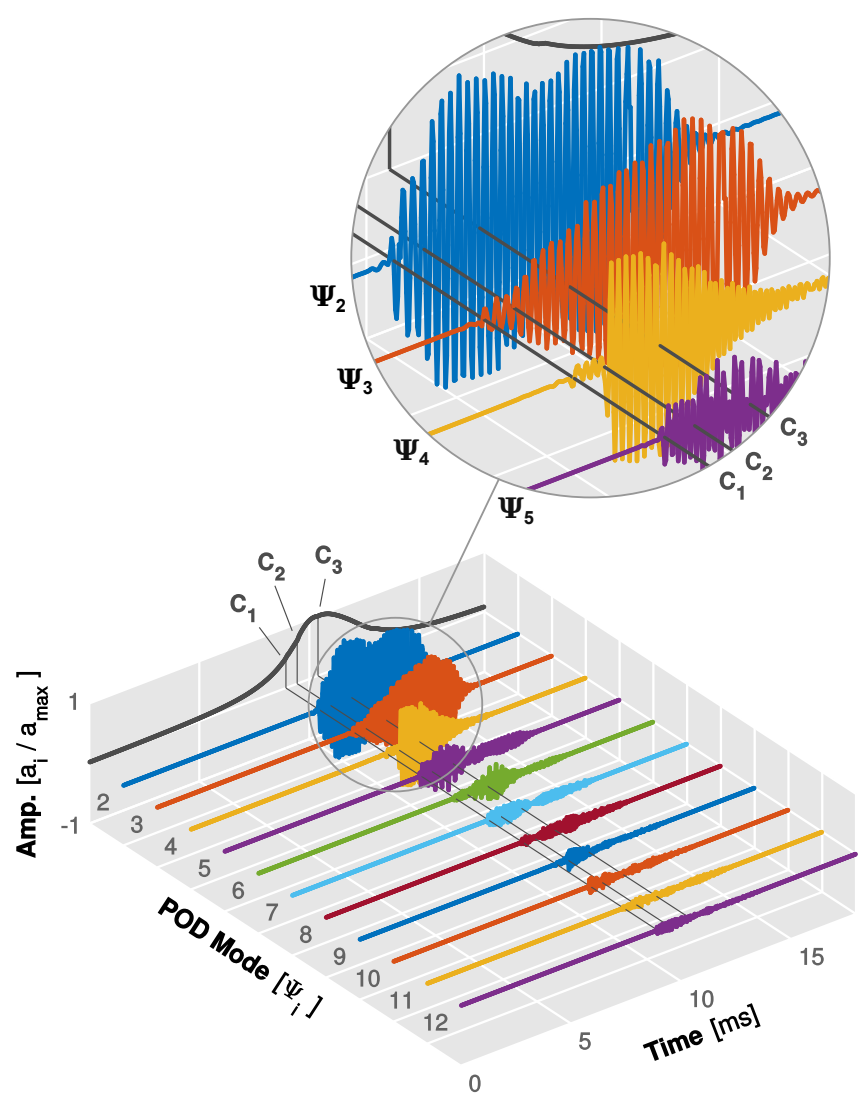

Figure 6: Normalized amplitude of POD modes $\boldsymbol{\Psi}_{1-12}$ in the time domain, based on normalized time coefficients $\mathbf{a}_{i}$. Ten percent fuel burning times $C_{i}$ for each of the three combustions are included for reference. 


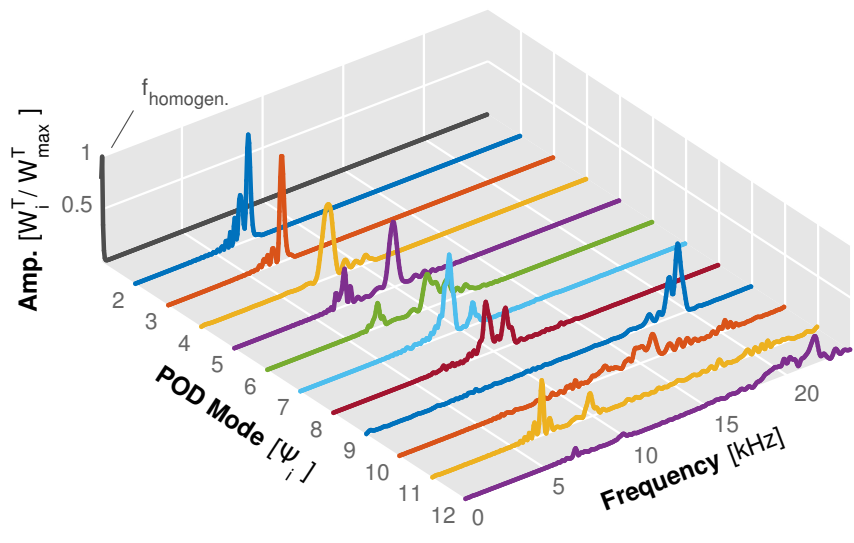

Figure 7: Normalized amplitude of POD modes $\boldsymbol{\Psi}_{1-12}$ in the frequency domain, based on normalized periodograms of rightsingular vectors $\mathbf{W}_{i}^{T}$. Scaling by singular values is avoided to allow a better visualization of the spectral content in higher order modes.

This coupling between the different burning times and the onset of different POD modes offers valuable insight into the acoustic effect of each separate fuel injection into the chamber, which will be useful when combined with the analysis of the spatial distribution of the modal energy.

Regarding now the frequency domain, periodograms in the case of Fig. 7 have been obtained from the right-singular vectors $\mathbf{W}^{\mathrm{T}}$ avoiding the scaling by $\sigma_{i}$, in order to better visualize the spectral content of the higher order modes that would appear flat if $\mathscr{F}\left\{\mathbf{a}_{i}\right\}$ had been used instead. Again, mode $\Psi_{1}$ is scaled independently from the rest, being clear in this figure that it contains the frequency peak corresponding to the compression-expansion process.

For the majority of reciprocating thermal engines, this mean pressure pulsation $\Psi_{1}$ is of very low frequency, and thus near or even below the human hearing threshold, diminishing its relevance when analyzing the acoustic output of the engine.

However, as seen in Fig. 7, the frequency content of the successive modes is concentrated well into the human hearing range, at $5-12 \mathrm{kHz}$ in this particular simulation. It can also be seen how, as previously mentioned, each POD mode can feature various frequencies of interest, making it difficult to ascribe a particular mode to a given phenomenon of known frequency, except in the case of $\boldsymbol{\Psi}_{1}$.

Finally, the spatial distribution of the POD modes can be inspected, by plotting the values of the left-singular vectors $\boldsymbol{\Psi}_{i}$ contained in the columns of $\mathbf{U}$ associated with each of the reference coordinates that were selected when building the snapshot matrix $\mathbf{V}$.

There are many ways of plotting the modal spatial information, such as slices at certain selected locations, isovolumes of a certain value, translucent volume rendering, etc. In Fig. 8, a set of isosurfaces is used to display POD modes $\Psi_{1-12}$, by showing the upper and lower $10 \%$ tails (this is, the $10 \%$ and $90 \%$ percentiles) of the distribution of their real values $\Re\left\{\boldsymbol{\Psi}_{i}\right\}$.
However, a previous step was necessary. The set of values $\left[\mathbf{x}^{\text {ref }}, \mathbf{y}^{\text {ref }}, \mathbf{z}^{\text {ref }}, \Re\left\{\boldsymbol{\Psi}_{i}\right\}\right]$ forms essentially a point cloud randomly distributed across the combustion chamber, lacking any connectivity information. This was achieved by performing a 3D Delaunay triangulation $[72,73]$ using the open-source application ParaView [74], which includes an $\alpha$-shape [75] related parameter to generate a more accurate reconstruction instead of just using the simple convex hull of the full dataset ${ }^{4}$, and then extracting two clips.

In this figure, red and blue volumes thus indicate the distribution of the top $10 \%$ positive and negative real values of the mode. As seen in Fig. 6 time coefficients $\mathbf{a}_{i}(t)$ take positive and negative values along the simulation, so if the mode shape was animated by plotting $\boldsymbol{\Psi}_{i} \mathbf{a}_{i}$ the red and blue volumes would identify the regions oscillating with alternating higher amplitudes. The only exception is $\boldsymbol{\Psi}_{i}$ which would display the homogeneous rise and fall in mean pressure.

Inspecting the shapes of modes $\Psi_{2}$ and $\Psi_{3}$ in Fig. 8 it is clearly seen how the higher amplitudes are oscillating on opposite sides of the squish zone, in two different orientations. Not depicted in the figure are the nodal regions whose amplitude remains constant in time, which in these two modes consist of a slice of the chamber aligned in the middle of the two colored isovolumes.

Furthermore, it can be seen that these two modes are reminiscent of classical acoustic transversal modes in opened combustion chambers, specifically mode $(m=1, n=0)$ in the notation of Hickling et al. [15], also called first asymmetric mode.

Continuing this comparison, POD mode $\boldsymbol{\Psi}_{4}$ features a completely circular distribution between the squish zone and the bowl, with an annular nodal region instead of a straight one like in the previous modes, being similar to Hickling's first radial mode $(m=0, n=1)$.

The next two modes $\boldsymbol{\Psi}_{5}$ and $\boldsymbol{\Psi}_{6}$ are interesting because they are remarkably three-dimensional. While they have show again a straight nodal area, regions of higher amplitudes are crossed between the bowl and the squish area, something that would not be visible if only $2 \mathrm{D}$ slices of the chamber were being considered in this analysis.

Modes $\boldsymbol{\Psi}_{7}$ and $\boldsymbol{\Psi}_{8}$ are again similar to classical transversal asymmetric modes, in this case related to mode $(m=2, n=0)$ shown by Hickling et al. Amplitudes in these two modes are again predominantly gathered in the squish area, but featuring two perpendicular nodal slices.

In contrast with previous modes, maximum amplitudes of $\boldsymbol{\Psi}_{9}$ are contained in the central region of the combustion chamber, alternating between the a single region located immediately over the center of the bowl and two opposite lower zones.

\footnotetext{
${ }^{4}$ Note that this $\alpha$ parameter, which limits the valid search distance to connect vertices, is dependant on the particular geometry and sparsity of the point cloud, but in any case it must be higher that $d_{c, \max }$.
} 


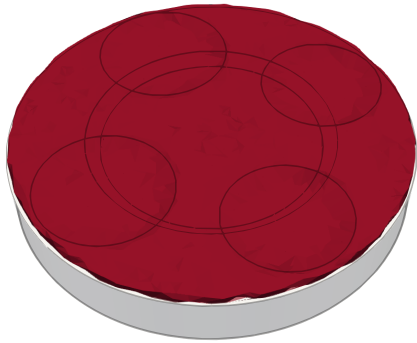

$\Psi_{1}$

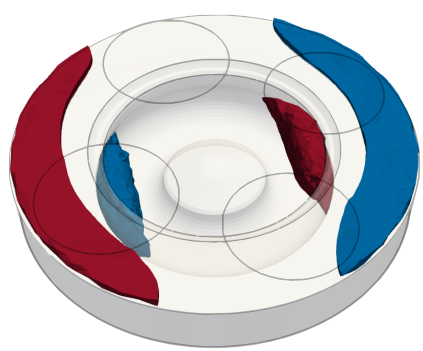

$\Psi_{5}$

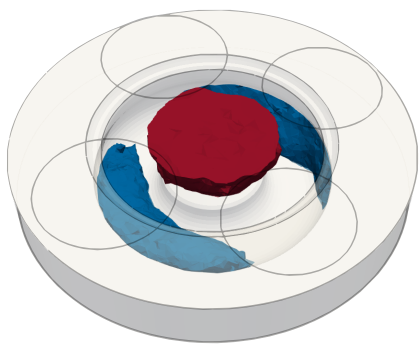

$\Psi_{9}$

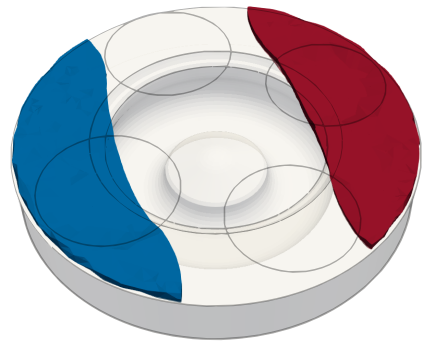

$\Psi_{2}$

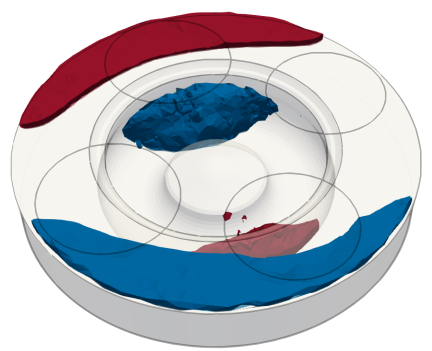

$\Psi_{6}$

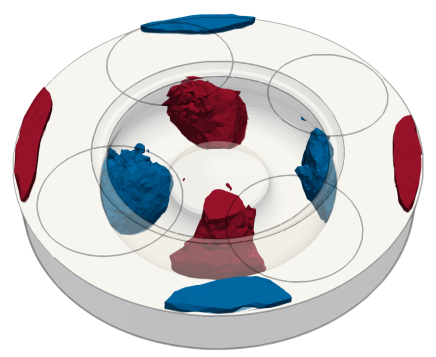

$\Psi_{10}$

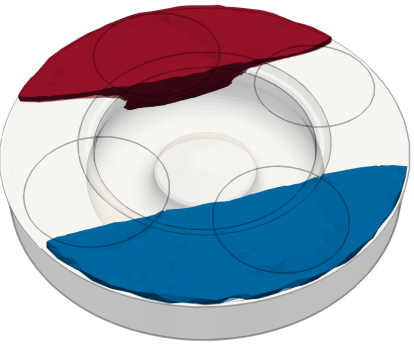

$\Psi_{3}$

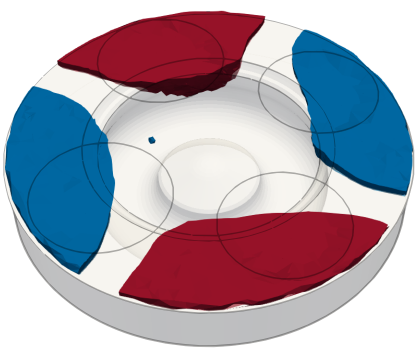

$\Psi_{7}$

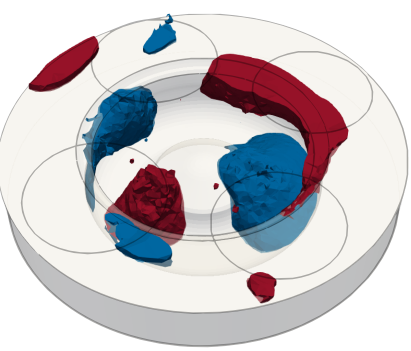

$\Psi_{11}$

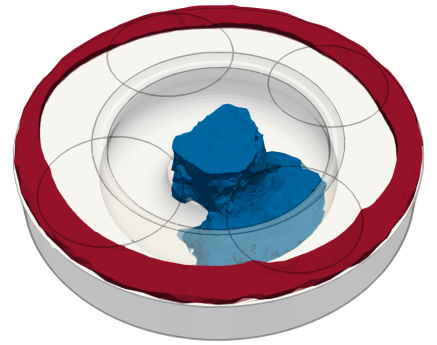

$\Psi_{4}$

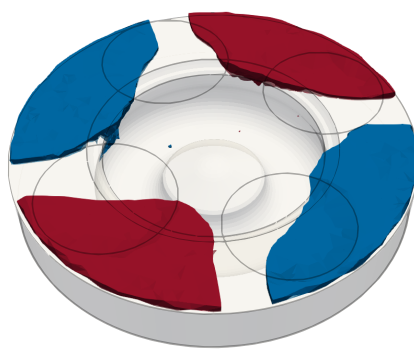

$\Psi_{8}$

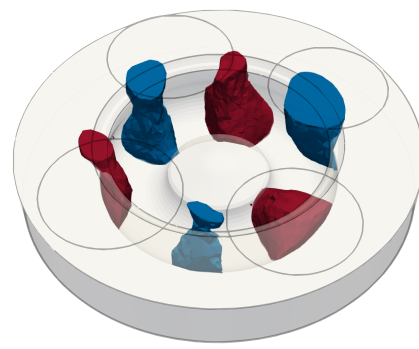

$\Psi_{12}$

Figure 8: Spatial distribution of POD modes $\Psi_{1-12}$ across the simulated combustion chamber. Each mode except for the homogeneously distributed $\Psi_{1}$ is represented by colored isovolumes indicating the 10\% (blue) and $90 \%$ (red) percentiles of the distribution of the real values of each individual mode $\Re\left\{\Psi_{i}\right\}$. The geometry of the piston head and the position of the valves are added for reference.

Recalling the time evolution of this node shown in Fig. 6, it can be seen how the frequency content of this particular mode, peaking around $19 \mathrm{kHz}$, is different from the previous squish-dominated modes in which the content was mainly spread between 6 and $11 \mathrm{kHz}$.

Subsequent mode $\boldsymbol{\Psi}_{10}$ presents again a pattern of mixed influence between the squish and the bowl contributions, in this case being similar to the previously discussed $\boldsymbol{\Psi}_{5}$ but with an added nodal slice as seen in modes $\boldsymbol{\Psi}_{7-8}$.

This mode, with bowl amplitudes again predominant, shows a frequency content centered around $15 \mathrm{kHz}$, thereby representing a transition from lower frequency squish-dominated modes and higher frequency bowl-dominated modes. Mode $\Psi_{11}$ also contains information in both regions but since the energy of these modes is already low, it appears to contain not-so-clear residual information.
Finally, high amplitude isovolumes in mode $\boldsymbol{\Psi}_{12}$ are again confined in the bowl, in this case exhibiting a three nodal slices pattern reminiscent of Hickling et al. transversal asymmetric mode ( $m=3, n=0)$. As it was the case with $\Psi_{9}$, this bowl-dominated mode contains mainly high frequency content gathered around $20 \mathrm{kHz}$.

\subsection{DMD decomposition}

As discussed in the theoretical background section, while it is possible to analyze the frequency content of POD modes and draw correlations between this content and the spatial distribution of each mode, Dynamic Mode Decomposition is a tool best suited for frequency analysis, as it forces each mode to contain a single frequency. 


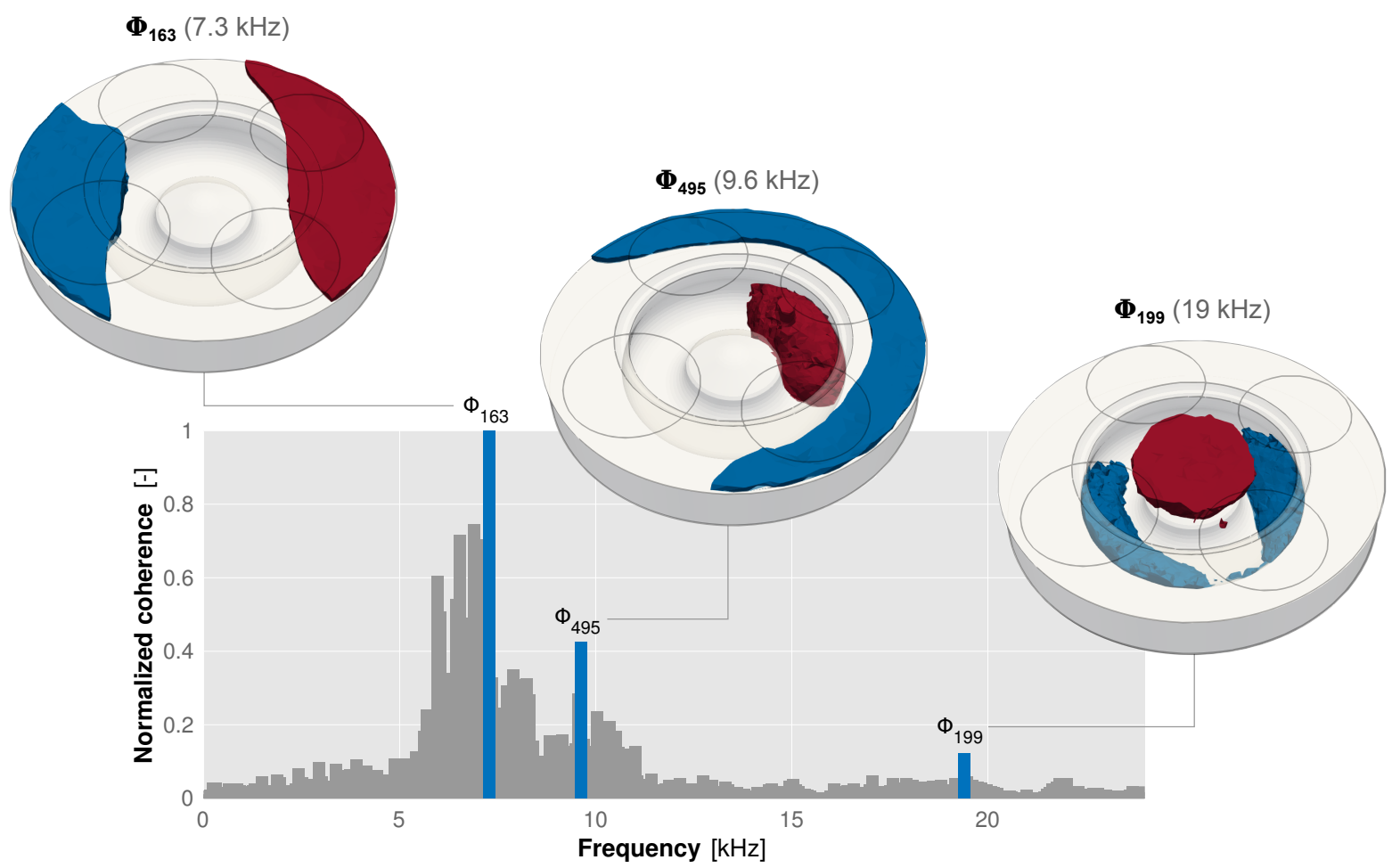

Figure 9: Normalized coherence $E_{i} / \max _{i}\left(E_{i}\right)$ of all DMD modes, highlighting the most coherent modes at the frequencies of interest: $\mathbf{\Phi}_{163}, \boldsymbol{\Phi}_{495}$ and $\mathbf{\Phi}_{199}$ and including their spatial distribution. Each mode is represented by colored isovolumes indicating the 10\% (blue) and $90 \%$ (red) percentiles of the distribution of the real values of each individual mode $\Re\left\{\boldsymbol{\Phi}_{i}\right\}$.

DMD was thus performed following the procedure outlined in section 2. In order to assess the relevance of the different resulting DMD modes $\boldsymbol{\Phi}_{i}$, the coherence $E_{i}$ of each mode was computed and then normalized with $\max _{i}\left(E_{i}\right)$. The magnitude of this metric for each mode can be seen in the chart of Fig. 9, where modes have been ordered according to their associated frequency.

It can be seen in this chart that three modes are peaking above those around them. Mode $\boldsymbol{\Phi}_{163}$ at around $7.3 \mathrm{kHz}$ is the most coherent node in the range of $5-8.5 \mathrm{kHz}, \boldsymbol{\Phi}_{495}$ is dominant among the $8.5-11 \mathrm{kHz}$ range and finally $\boldsymbol{\Phi}_{199}$ appears as a single peaking mode at $19 \mathrm{kHz}$. The spatial distributions of these three modes have been included in Fig. 9 following the same procedure used in the POD case.

These spatial distributions along with the modal coherence information confirms and validates the correlations between frequency content and spatial distributions that were already suggested by the POD total energy ranking: the first group of frequencies, exemplified by $\boldsymbol{\Phi}_{163}$, is the dominant in this combustion simulation, and it is clearly dominated by the reflections in the squish zone.

A second, smaller set of slightly higher frequency content is represented by mode $\boldsymbol{\Phi}_{495}$, which is related to the interaction of the pressure fluctuations between the squish zone and the bowl. Finally, higher frequency content near $20 \mathrm{kHz}$ is linked to the pressure fluctuations contained within the bowl, as shown by the spatial distribution of DMD mode $\boldsymbol{\Phi}_{199}$.

\section{Practical application}

The conclusions offered by both decomposition procedures about the correlation between spatial, temporal and frequency content of the unsteady flow field behaviour in the chamber can be applied to the practical optimization of the combustion process, in order to mitigate the noise emission of the engine.

As seen in Fig. 9, in this combustion acoustic energy is mainly gathered in the $6-11 \mathrm{kHz}$ band, which is coincidentally favoured by human hearing. An optimization strategy could consist in trying to demote this content shifting the acoustic energy to higher frequencies, which are naturally attenuated by the human auditory system. ITU-R BS.468-4 standard for instance quantifies this attenuation in $20.2 \mathrm{~dB}$ at $20 \mathrm{kHz}[76]$.

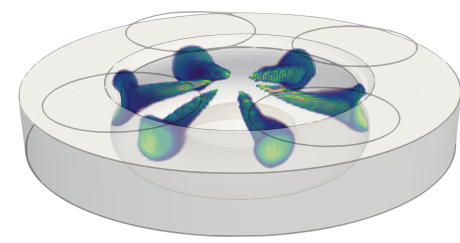

Original $\left[150^{\circ}\right]$

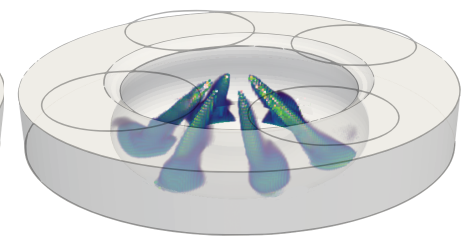

Modified $\left[90^{\circ}\right]$
Figure 10: Original and modified sprays visualized as a clip of equivalence ratio $\phi>1$ at approximately 7 CAD after top dead center in both cases. 
Thus, if the energy represented by DMD modes $\boldsymbol{\Phi}_{163}$ and $\boldsymbol{\Phi}_{495}$ could be shifted towards mode $\boldsymbol{\Phi}_{199}$ or, even better, independently reduced, the overall perceived noise level would be reduced. In order to do this, the information gained by means of the decomposition techniques can prove of utility.

Since it has been established that the $6-11 \mathrm{kHz}$ content is mainly linked to the excitation in the squish area of the chamber, which is in turn caused by the location of the combustion energy release, a way to alter this dynamic behaviour could be to modify the angle of the fuel injection, directing the sprays slightly more towards the piston in order to promote bowl-dominated content against squish-dominated content.

To test this approach, another CFD simulation was carried out where the included spray angle was modified from $150^{\circ}$ to $90^{\circ}$, keeping constant the remaining combustion parameters and following the same procedure as outlined in section 3 .

In Fig. 10 it is shown how the modified angle changes the impingement points of the sprays from the upper part of the bowl and the start of the squish in the original case, to near the bottom of the bowl wall in the $90^{\circ}$ case.

The sprays are visualized in this figure by clipping the equivalence ratio $\phi$ at the unitary value, thereby showing the isovolume of stoichiometric conditions enclosing the spray. If $C, H$ and $O$ are the number of carbon, hydrogen, and oxygen atoms in a cell, the equivalence ratio is computed as:

$$
\phi=\frac{2 C+H / 2}{O}
$$

In order to understand how the spatial distribution of the unsteady pressure fluctuations has been affected by the new spray angle, POD was performed on the modified CFD results. Fig. 11 shows how the modal energy has shifted from the original to the modified combustion.

The energy share and spatial distribution of modified POD modes $\widetilde{\Psi}_{2-5}$ are plotted, along with that of the most closely resembling original mode. Again, mode $\widetilde{\boldsymbol{\Psi}}_{1}$ is disregarded since it is only related to the homogeneous compression-expansion pressure cycle. It can be seen how the circular $(m=0, n=1)$ mode which was previously ranked fourth with $11.2 \%$ of the energy, is now the most relevant with an energy share of $18.25 \%$.

Modified modes $\widetilde{\Psi}_{3-4}$ are shown to closely resemble the original $\Psi_{2-3}$ modes, albeit with a rotation of the nodal plane, which is probably due to a different spray igniting first and thus initiating these squish-dominated propagating pressure oscillations. Their energy contents on the other hand have been diminished to less than half their previous ones. Lastly, the original bowl-dominated mode $\boldsymbol{\Psi}_{9}$ has been promoted to the fourth place with almost four times its previous energy.

To show how this shifting in the modal energy has affected the audible content, in Fig. 12 a comparison between the acoustic signature of the original and the modified combustions can be found, where the averaged Sound Pressure Level (SPL) across the chamber has been plotted. Decibel scale has been selected in order to better assess the difference at each frequency, and the bands used in Fig. 4 have been kept for reference.

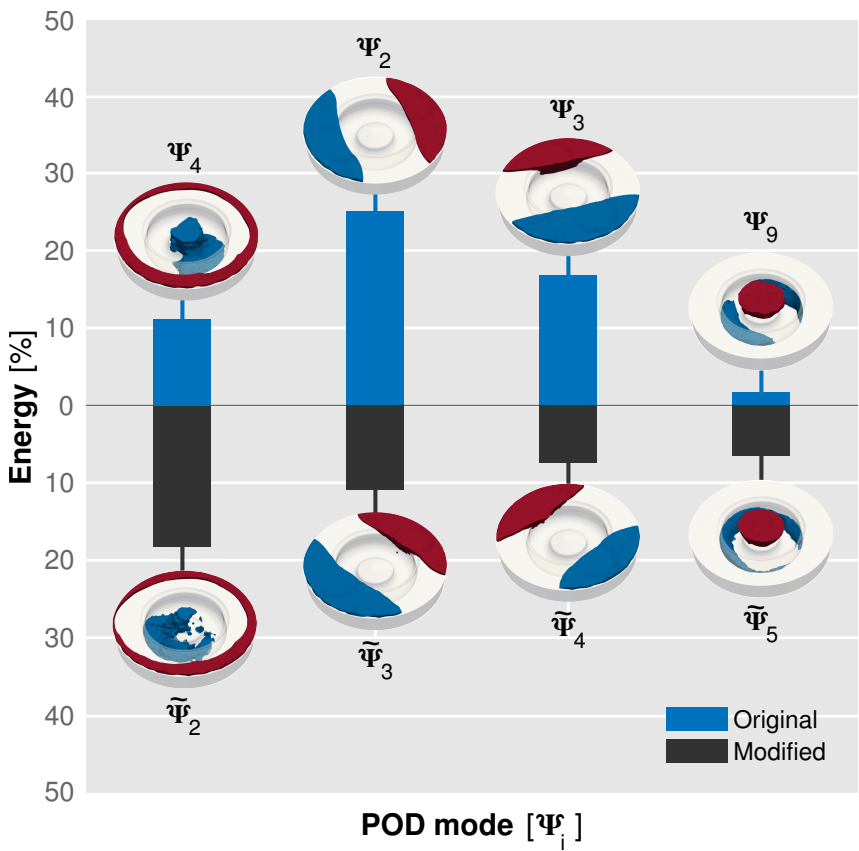

Figure 11: Energy share and spatial distribution of the four most relevant modified modes $\widetilde{\Psi}_{2-5}$ (bottom), together with their most closely resembling original counterparts (top).

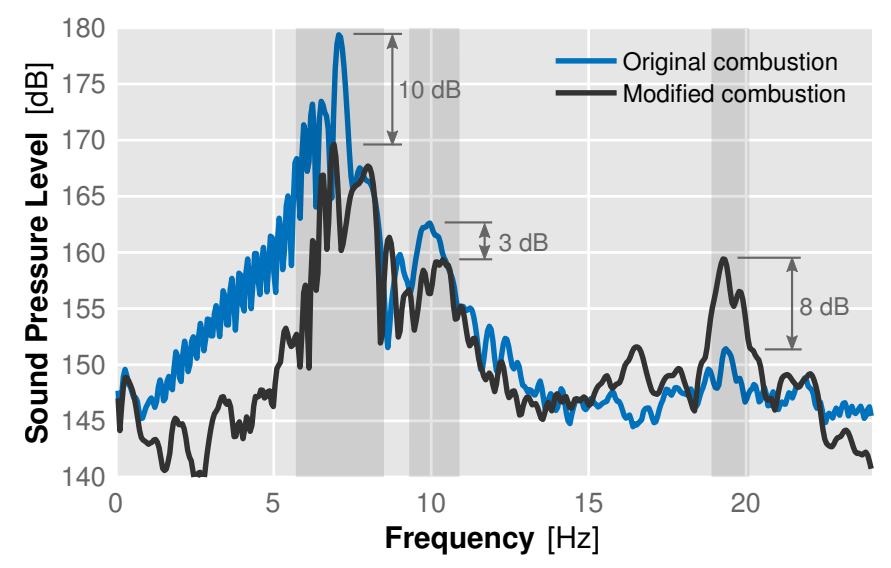

Figure 12: Comparison between the averaged Sound Pressure Level (SPL) of the original simulated combustion cycle and the SPL of the modified combustion with spray angle $90^{\circ}$.

As predicted by the modal decomposition results, noise levels at squish-dominated frequencies $(6-8.5 \mathrm{kHz})$ are significantly reduced, by approximately $10 \mathrm{~dB}$. Acoustic content in the $9-11 \mathrm{kHz}$ band where DMD decomposition attested mixed influence of the squish zone and the bowl is also reduced, although not as much as in the previous band.

On the contrary, and again as predicted, the shifting of the combustion excitation towards the bowl caused indeed an appreciable increase in the $19 \mathrm{kHz}$ acoustic content which is linked to this zone of the chamber. Still, the natural human hearing attenuation mitigates the relevance of this increase since the content at this frequency is still masked by the medium-frequency noise emission. 
It is true that this optimization of acoustic emission should not be performed on its own, as the possible increase in chemical pollutants and decrease of combustion efficiency is affected as well by the angle of the injection. However, modal decomposition is shown to provide valuable information to better initialize and define a merit function for an optimization algorithm.

\section{Conclusions}

In this paper, a numerical model of a representative CI combustion chamber has been considered in order to characterize the unsteady behaviour caused by the combustion process. Experimental validation of this numerical model has been conducted, ensuring that a correct representation of the pressure evolution within the combustion cycle is achieved.

A methodology has been proposed to extract coherent time-spatial data from this simulation, even in cases where the mesh is changing due to moving components (cylinders, valves) or refining-coarsening optimizations designed to reduce the computation time.

Pressure data assembled in this way was postprocessed using different techniques. A Fourier analysis was performed where the FFT of each cell was computed, and spatial features were extracted, filtering certain frequency ranges of interest selected manually based on idealized modes expected from the literature.

In order to automate this task and also to obtain a deeper insight into the time-spatial modal characteristics of the combustion unsteady flow field, POD was applied to the same pressure data. Clear three-dimensional pressure modes have been identified using this technique, reminiscent of classical open chambers acoustic modes but demonstrating for the first time the additional complex interaction of the pressure waves in the different zones in the chamber geometry.

Time evolution of these POD modes has been shown to correlate with the different phases of the combustion cycle, showing for instance how the annular resonance of the chamber is caused by the combustion of the second injection, thereby offering greater insight into the contribution of timing settings and geometric parameters to the overall acoustic resonance of the chamber by decoupling both domains time and spatial domains.

Another advantage of POD modes is their dimensionalityreduction optimality, allowing the pressure signals to be adequately reconstructed with only a low number of modes. In particular, results of this paper show that $50 \%$ of the combustion acoustic energy is contained in just 4 out of the 862 modes that were computed, while $99 \%$ is contained in the first 180 modes, indicating that $99 \%$ accurate characterization of the pressure field is possible with almost 8 times less data storage, which is an important constraint of large numerical simulations. Also, as suggested by Druault [26], these reduced modes can be useful for initialising LES models.
This optimum representation however implies that each mode carries a mixed frequency content. If the detailed analysis of the frequency domain is intended, or if identification of low-energy, short-lived modes is sought, DMD can be preferred to POD, as it was introduced to overcome these shortcomings. In this case, DMD has validated what POD already indicated: spectral content at $5-8.5 \mathrm{kHz}$ is related to the squish-dominated pulsation, with the $8.5-11 \mathrm{kHz}$ featuring squish-bowl interaction and the higher frequency content at $19 \mathrm{kHz}$ being related to central top-down bowl oscillations.

Advantage was taken of this spatial-frequency decomposition of the combustion to showcase a practical application where the spray angle was redirected in order to promote higher frequency bowl-dominated modes, since results have established these modes to feature higher frequency content, less perceptible by the human hearing.

POD decomposition of this modified combustion was performed, revealing the energy shifting between modes as a result of the different pressure excitation location. The annular mode was promoted from third to first in combustion acoustic relevance, with the bowl-dominated mode almost quadrupling its energy share. Squish-dominated modal energy was however almost halved.

The intended effect of the modified modal content on the averaged acoustic levels of the combustion chamber was also validated, exhibiting a reduction of the sensitive midfrequency content, at the cost of an increase in less adverse higher frequency content.

This result highlights the advantages of the enhanced understanding of combustion chambers' time-spatial unsteady structures obtained in this work through modal decomposition techniques. Specifically, combustion acoustics can be improved by identifying and tackling the most relevant modes, allowing automated optimization algorithms to modify the combustion settings and/or chamber geometries in a more meaningful way, pursuant to the final objective of addressing the noise emission challenge faced by thermal-powered vehicles.

\section{Acknowledgements}

The equipment used in this work has been partially supported by FEDER project funds "Dotación de infraestructuras científico técnicas para el Centro Integral de Mejora Energética y Medioambiental de Sistemas de Transporte (CiMeT)" [grant number FEDER-ICTS-2012-06], framed in the operational program of unique scientific and technical infrastructure of the Spanish Government.

J. Gomez-Soriano is partially supported through the Programa de Apoyo para la Investigación y Desarrollo (PAID) of Universitat Politècnica de València [grant number FPI-S22016-1353]. J. García-Tíscar is partially supported through the Programa de Apoyo para la Investigación y Desarrollo (PAID) of Universitat Politècnica de València [grant number FPI-S2-2015-1530]. 
The authors also want to express their gratitude to CONVERGENT SCIENCE Inc. and IGNITE3D Engineering GmbH for their kind support in performing the CFD calculations using CONVERGE software.

\section{References}

[1] M. Guarnieri, Looking back to electric cars, in: History of Electrotechnology Conference (HISTELCON), IEEE, 2012, pp. 1-6. doi : 10 1109/HISTELCON . 2012 . 6487583.

[2] B. Masterton, H. Heffner, R. Ravizza, The evolution of human hearing, The J. Acoust. Soc. Am. 45 (4) (1969) 966-985. doi:10.1121/1. 1911574.

[3] A. J. Torregrosa, A. Broatch, J. Martín, L. Monelletta, Combustion noise level assessment in direct injection diesel engines by means of in-cylinder pressure components, Meas Sci Technol 18 (7) (2007) 21312142. doi:doi: 10.1088/0957-0233/18/7/045.

[4] A. de Risi, T. Donateo, D. Laforgia, Optimization of the combustion chamber of direct injection diesel engines, in: SAE Technical Paper, SAE International, 2003. doi : 10.4271/2003-01- 1064.

[5] J. Benajes, R. Novella, J. M. Pastor, A. Hernández-López, M. Hasegawa, N. Tsuji, M. Emi, I. Uehara, J. Martorell, M. Alonso, Optimization of the combustion system of a medium duty direct injection diesel engine by combining $\{C F D\}$ modeling with experimental validation, Energy Convers. Manage. 110 (2016) 212 - 229. doi : 10. 1016/j . enconman . 2015.12 .010

[6] H. Hiroyasu, H. Miao, T. Hiroyasu, M. Miki, J. Kamiura, S. Watanabe, Genetic algorithms optimization of diesel engine emissions and fuel efficiency with air swirl, EGR, injection timing and multiple injections, in: SAE Technical Paper, SAE International, 2003. doi: 10. 4271/2003-01- 1853.

[7] M. Costa, G. M. Bianchi, C. Forte, G. Cazzoli, A numerical methodology for the multi-objective optimization of the DI diesel engine combustion, Energy Procedia 45 (2014) $711-720$. doi: 10.1016/j . egypro. 2014. 01.076 .

[8] A. J. Torregrosa, A. Broatch, X. Margot, V. Marant, Combustion chamber resonances in direct injection automotive Diesel engines: a numerical approach, InterNatl. J. (Wash.) of Engine Research 5 (1) (2003) 83-91. doi: $10.1243 / 146808704772914264$.

[9] M. Wissink, Z. Wang, D. Splitter, A. Shahlari, R. D. Reitz, Investigation of pressure oscillation modes and audible noise in RCCI, HCCI, and CDC, in: SAE Technical Paper, Vol. 2013-01-1652, SAE International, 2013. doi: 10.4271/2013-01-1652.

[10] A. Broatch, X. Margot, A. Gil, C. Donayre, Computational study of the sensitivity to ignition characteristics of the resonance in DI diesel engine combustion chambers, Engineering Computations 24 (1) (2007) 77-96. doi: 10.1108/02644400710718583.

[11] A. Broatch, X. Margot, R. Novella, J. Gomez-Soriano, Impact of the injector design on the combustion noise of gasoline partially premixed combustion in a 2-stroke engine, Applied Therm. Eng. 119 (2017) 530 -540. doi:10.1016/j .applthermaleng.2017.03.081.

[12] C. Draper, Pressure waves accompanying detonation in the internal combustion engine, J. Aeronaut. Sci. 5 (6) (1938) 219-226. doi: 10.2514/8.590.

[13] T. Priede, Relation between form of cylinder-pressure diagram and noise in diesel engines, Proceedings of the Institution of Mechanical Engineers: Automobile Division 14 (1) (1960) 63-97. doi:10.1243/ pime_auto_1960_000_012_02.

[14] T. Priede, E. Grover, Paper 2: Noise of industrial diesel engines, in: Proceedings of the Institution of Mechanical Engineers, Conference Proceedings, Vol. 181, SAGE Publications Sage UK: London, England, 1966, pp. 73-89. doi:10.1243/pime_conf_1966_181_062_02.

[15] R. Hickling, D. A. Feldmaier, S. H. Sung, Knock-induced cavity resonances in open chamber diesel engines, The J. Acoust. Soc. Am. 65 (6) (1979) 1474-1479. doi:10.1121/1.382910.

[16] Y. Liang, H. Lee, S. Lim, W. Lin, K. Lee, C. Wu, Proper orthogonal decomposition and its applications-part i: Theory, J. Sound Vib. 252 (3) (2002) 527-544. doi:10.1006/jsvi.2001.4041.
[17] J. L. Lumley, The structure of inhomogeneous turbulent flows, in: Atmospheric Turbulence and Radio Wave Propagation - Proceedings of the International Colloquium, Nauka, Moscow, 1967, pp. 166-178.

[18] N. Aubry, On the hidden beauty of the proper orthogonal decomposition, Theor. Comput. Fluid Dyn. 2 (5-6) (1991) 339-352. doi: 10.1007/ BF00271473.

[19] P. J. Schmid, Dynamic mode decomposition of numerical and experimental data, J Fluid Mech 656 (2010) 5-28. doi:10.1017/ S0022112010001217.

[20] S. Bagheri, Koopman-mode decomposition of the cylinder wake, J Fluid Mech 726 (2013) 596-623. doi : 10.1017/j fm.2013.249.

[21] S. Wold, K. Esbensen, P. Geladi, Principal component analysis, Chemom Intell Lab Syst 2 (1-3) (1987) 37-52. doi: 10. 1016/0169-7439(87) 80084- 9.

[22] H. Abdi, L. J. Williams, Principal component analysis, Wiley Interdiscip. Rev. Comput. Stat. 2 (4) (2010) 433-459. doi:10.1002/wics. 101.

[23] V. Nikiforov, The energy of graphs and matrices, J. Math. Analysis Appl. 326 (2) (2007) 1472-1475. doi:10.1016/j . jmaa. 2006 .03.072.

[24] C. Eckart, G. Young, The approximation of one matrix by another of lower rank, Psychometrika 1 (3) (1936) 211-218. doi:10.1007/ BFO2288367.

[25] P. Druault, J. Delville, J.-P. Bonnet, Proper orthogonal decomposition of the mixing layer flow into coherent structures and turbulent gaussian fluctuations, C.R. Mec. 333 (11) (2005) 824-829. doi:10.1016/j. crme.2005.10.001.

[26] P. Druault, S. Lardeau, J. Bonnet, F. Coiffet, J. Delville, E. Lamballais, J. Largeau, L. Perret, Generation of three-dimensional turbulent inlet conditions for large-eddy simulation, AIAA journal 42 (3) (2004) 447456. doi: $10.2514 / 1.3946$.

[27] P. J. Schmid, L. Li, M. P. Juniper, O. Pust, Applications of the dynamic mode decomposition, Theor. Comput. Fluid Dyn. 25 (1-4) (2011) 249259. doi : 10.1007/s00162-010-0203-9.

[28] M. R. Jovanović, P. J. Schmid, J. W. Nichols, Sparsity-promoting dynamic mode decomposition, Phys. Fluids (1994-present) 26 (2) (2014) 024103. doi: 10.1063/1.4863670.

[29] A. Sakowitz, M. Mihaescu, L. Fuchs, Flow decomposition methods applied to the flow in an ic engine manifold, Applied Therm. Eng. 65 (1) (2014) 57-65. doi:10.1016/j . applthermaleng . 2013.12.082.

[30] Y. Huang, V. Yang, Dynamics and stability of lean-premixed swirlstabilized combustion, Prog. Energy Combust. Sci. 35 (4) (2009) 293364. doi:10.1016/j.pecs. 2009.01.002.

[31] A. M. Steinberg, I. Boxx, M. Stöhr, C. D. Carter, W. Meier, Flow-flame interactions causing acoustically coupled heat release fluctuations in a thermo-acoustically unstable gas turbine model combustor, Combust. Flame 157 (12) (2010) 2250-2266. doi:10.1016/j . combustflame. 2010.07.011.

[32] V. Caux-Brisebois, A. M. Steinberg, C. M. Arndt, W. Meier, Thermoacoustic velocity coupling in a swirl stabilized gas turbine model combustor, Combust. Flame 161 (12) (2014) 3166-3180. doi:10.1016/j . combustflame. 2014.05.020.

[33] J. Meadows, A. K. Agrawal, Time-resolved piv of lean premixed combustion without and with porous inert media for acoustic control, Combust. Flame 162 (4) (2015) 1063-1077. doi:10.1016/j.combust flame. 2014.09. 028.

[34] M. Fogleman, J. Lumley, D. Rempfer, D. Haworth, Application of the proper orthogonal decomposition to datasets of internal combustion engine flows., J. Turbul. 5 (23) (2004) 1-3. doi : 10. 1088/1468 - 5248/ 5/1/023.

[35] S. Kostka, A. C. Lynch, B. C. Huelskamp, B. V. Kiel, J. R. Gord, S. Roy, Characterization of flame-shedding behavior behind a bluff-body using proper orthogonal decomposition, Combust. Flame 159 (9) (2012) 2872-2882. doi:10.1016/j . combust flame. 2012.03.021.

[36] K. Bizon, G. Continillo, E. Mancaruso, S. Merola, B. Vaglieco, Pod-based analysis of combustion images in optically accessible engines, Combust. Flame 157 (4) (2010) 632-640. doi: 10.1016/j . combust flame. 2009. 12.013.

[37] K. Bizon, G. Continillo, S. Lombardi, P. Sementa, B. M. Vaglieco, Independent component analysis of cycle resolved combustion images from a spark ignition optical engine, Combust. Flame 163 (2016) 258-269. doi:10.1016/j . combustflame.2015.10.002. 
[38] H. Chen, D. L. Hung, M. Xu, H. Zhuang, J. Yang, Proper orthogonal decomposition analysis of fuel spray structure variation in a sparkignition direct-injection optical engine, Exp. Fluids 55 (4) (2014) 1703. doi : 10.1007/s00348-014-1703-y.

[39] H. Chen, D. L. Reuss, V. Sick, Analysis of misfires in a direct injection engine using proper orthogonal decomposition, Exp. Fluids 51 (4) (2011) 1139-1151. doi: 10.1007/s00348-011-1133-z.

[40] S. J. Danby, T. Echekki, Proper orthogonal decomposition analysis of autoignition simulation data of nonhomogeneous hydrogen-air mixtures, Combust. Flame 144 (1) (2006) 126-138. doi:10.1016/j. combustflame.2005.06.014.

[41] C. W. Rowley, I. Mezić, S. Bagheri, P. Schlatter, D. S. Henningson, Spectral analysis of nonlinear flows, J Fluid Mech 641 (2009) 115-127. doi : 10.1017/S0022112009992059.

[42] K. K. Chen, J. H. Tu, C. W. Rowley, Variants of dynamic mode decomposition: boundary condition, koopman, and fourier analyses, J. Non-linear Sci. 22 (6) (2012) 887-915. doi : 10. 1007/s00332-012-9130- 9.

[43] F. Richecoeur, L. Hakim, A. Renaud, L. Zimmer, DMD algorithms for experimental data processing in combustion, in: Proceedings of the Summer Program 2012, Center for Turbulence Research, Stanford University, 2012, pp. 459-468.

URL https://web.stanford.edu/group/ctr/Summer/SP12/06.08_ richecouer.pdf

[44] R. Sampath, S. R. Chakravarthy, Investigation of intermittent oscillations in a premixed dump combustor using time-resolved particle image velocimetry, Combust. Flame 172 (2016) 309-325. doi: 10. 1016/j . combustflame. 2016.06.018.

[45] B. O. Koopman, Hamiltonian systems and transformation in hilbert space, Proc. Natl. Acad. Sci. 17 (5) (1931) 315-318. URL http://www. pnas . org/content/17/5/315. full.pdf

[46] T. Sayadi, J. Nichols, P. Schmid, M. Jovanovic, Dynamic mode decomposition of h-type transition to turbulence, in: Proceedings of the Summer Program 2012, Center for Turbulence Research, Stanford University, 2012, pp. 5-14.

URL http://people.ece.umn.edu/users/mihailo/papers/ conference/2012/SayadiNicholsSchmidJovanovicCTR2012. pdf

[47] J. Dahan, R. Futrzynski, C. O’Reilly, G. Efraimsson, Aero-acoustic source analysis of landing gear noise via dynamic mode decomposition, in: 21st International Congress on Sound and Vibration, 2014.

URL http://www.iiav.org/icsv21/content/papers/papers/ full_paper_221_20140402145649114.pdf

[48] R. Futrzynski, G. Efraimsson, Dymode: A parallel dynamic mode decomposition software, KTH Royal Institute of Technology (2015). URL http://kth.diva-portal.org/smash/record.jsf?pid= diva2\%3A786623

[49] Y. T. Delorme, A.-E. M. Kerlo, K. Anupindi, M. D. Rodefeld, S. H. Frankel, Dynamic mode decomposition of fontan hemodynamics in an idealized total cavopulmonary connection, Fluid Dyn. Res. 46 (4) (2014) 041425. doi : 10. 1088/0169-5983/46/4/041425.

[50] J. F. Williams, D. L. Hawkings, Sound generation by turbulence and surfaces in arbitrary motion, Philos. Trans. Roy. Soc. London A: Mathematical, Physical and Eng. Sci. 264 (1151) (1969) 321-342. doi: 10.1098/rsta. 1969.0031.

[51] V. Yakhot, S. Orszag, Renormalization group analysis of turbulence., J. Sci. Comput. 1 (1) (1986) 3-51. doi : doi : 10. 1007/BF01061452.

[52] C. Angelberger, T. Poinsot, B. Delhay, Improving near-wall combustion and wall heat transfer modeling in si engine computations, in: SAE Technical Paper, SAE International, 1997. doi : 10.4271/972881. URL 10.4271/972881

[53] O. Redlich, J. N. S. Kwong, On the thermodynamics of solutions. V. An equation of state. Fugacities of gaseous solutions, Chem Rev 44 (1) (1949) 233-244. doi:10.1021/cr60137a013.

[54] R. Issa, Solution of the implicitly discretised fluid flow equations by operator-splitting, J Comput Phys 62 (1986) 40-65. doi:10.1016/ 0021 - 9991 ( 86 ) 90099 - 9.

[55] J. Würmel, J. Simmie, Cfd studies of a twin-piston rapid compression machine, Combust. Flame 141 (4) (2005) 417-430. doi: 10.1016/j combustflame.2005.01.015.
[56] S. Som, S. Aggarwal, Effects of primary breakup modeling on spray and combustion characteristics of compression ignition engines, Combust. Flame 157 (6) (2010) 1179-1193. doi:10.1016/j.combustflame. 2010.02 .018$.

[57] A. A. Moiz, M. M. Ameen, S.-Y. Lee, S. Som, Study of soot production for double injections of n-dodecane in ci engine-like conditions, Combust. Flame 173 (2016) 123-131. doi:10.1016/j.combustflame.2016. 08.005.

[58] Y. M. Wright, O.-N. Margari, K. Boulouchos, G. De Paola, E. Mastorakos, Experiments and simulations of n-heptane spray auto-ignition in a closed combustion chamber at diesel engine conditions, Flow, turbulence and combustion 84 (1) (2010) 49-78. doi:10.1007/ s10494- 009- $9224-0$.

[59] Z. Han, R. D. Reitz, Turbulence modeling of internal combustion engines using rng $k-\varepsilon$ models, Combust. Sci. Technol. 106 (4-6) (1995) 267295. doi : 10. 1080/00102209508907782.

[60] J. K. Dukowicz, A particle-fluid numerical model for liquid sprays, J Comput Phys 35 (2) (1980) 229 - 253. doi : 10. 1016/0021-9991(80) $90087-X$.

[61] R. D. Reitz, J. C. Beale, Modeling spray atomization with the kelvinhelmholtz/rayleigh-taylor hybrid model, Atomization Sprays 9 (6) (1999) 623-650.

[62] P. K. Senecal, E. Pomraning, K. J. Richards, T. E. Briggs, C. Y. Choi, R. M. McDavid, M. A. Patterson, Multi-dimensional modeling of directinjection diesel spray liquid length and flame lift-off length using cfd and parallel detailed chemistry, in: SAE Technical Paper, SAE International, 2003. doi : 10.4271/2003-01- 1043.

[63] J. Brakora, R. D. Reitz, A comprehensive combustion model for biodiesel-fueled engine simulations, in: SAE Technical Paper, SAE International, 2013. doi : 10.4271/2013-01- 1099.

[64] A. Torregrosa, P. Olmeda, B. Degraeuwe, M. Reyes, A concise wall temperature model for DI diesel engines, Applied Therm. Eng. 26 (11-12) (2006) 1320-1327. doi : 10.1016/j .applthermaleng. 2005.10.021.

[65] F. Payri, P. Olmeda, J. Martín, A. García, A complete OD thermodynamic predictive model for direct injection diesel engines, Appl. Energy 88 (12) (2011) 4632-4641. doi : 10 . 1016/j . apenergy . 2011.06.005.

[66] A. Broatch, X. Margot, R. Novella, J. Gomez-Soriano, Combustion noise analysis of partially premixed combustion concept using gasoline fuel in a 2-stroke engine, Energy 107 (2016) 612-624. doi:10.1016/j . energy.2016.04.045.

[67] A. E. W. Austen, T. Priede, Origins of Diesel engine noise, in: SAE Technical Paper, SAE International, 1959. doi : 10.4271/590127.

[68] W. C. Strahle, Combustion randomness and diesel engine noise: theory and initial experiments, Combust. Flame 28 (1977) 279-290. doi: 10. 1016/0010-2180(77) 90033- 5 .

[69] O. Vermorel, S. Richard, O. Colin, C. Angelberger, A. Benkenida, D. Veynante, Towards the understanding of cyclic variability in a spark ignited engine using multi-cycle les, Combust. Flame 156 (8) (2009) 15251541. doi:10.1016/j . combustflame . 2009.04 .007.

[70] K. Truffin, C. Angelberger, S. Richard, C. Pera, Using large-eddy simulation and multivariate analysis to understand the sources of combustion cyclic variability in a spark-ignition engine, Combust. Flame 162 (12) (2015) 4371-4390. doi : 10.1016/j . combustflame. 2015.07.003.

[71] A. Misdariis, O. Vermorel, T. Poinsot, Les of knocking in engines using dual heat transfer and two-step reduced schemes, Combust. Flame 162 (11) (2015) 4304-4312. doi:10.1016/j . combustflame. 2015. 07.023.

[72] J. R. Shewchuk, Tetrahedral mesh generation by delaunay refinement, in: Proceedings of the fourteenth annual symposium on Computational geometry, ACM, 1998, pp. 86-95. doi : 10.1145/276884.276894.

[73] L. P. Chew, Guaranteed-quality delaunay meshing in 3d (short version), in: Proceedings of the thirteenth annual symposium on Computational geometry, ACM, 1997, pp. 391-393. doi : 10.1145/262839.263018.

[74] A. Henderson, J. Ahrens, C. Law, et al., The ParaView Guide, Kitware Clifton Park, NY, 2004.

[75] H. Edelsbrunner, D. Kirkpatrick, R. Seidel, On the shape of a set of points in the plane 29 (4) (1983) 551-559. doi:10.1109/TIT. 1983. 1056714.

[76] ITU, BS.468: Measurement of audio-frequency noise voltage level in sound broadcasting (1986).

URL https : //Www. itu. int/rec/R-REC- BS . 468 - 4 - 198607 - I/en 